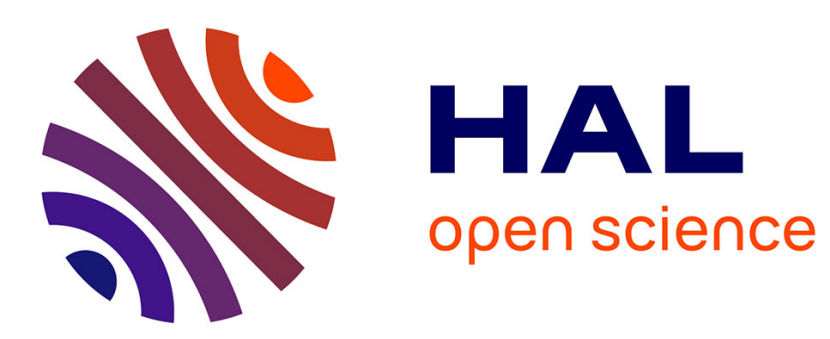

\title{
An ALE formulation for explicit Runge-Kutta Residual Distribution
}

Luca Arpaia, Mario Ricchiuto, Remi Abgrall

\section{To cite this version:}

Luca Arpaia, Mario Ricchiuto, Remi Abgrall. An ALE formulation for explicit Runge-Kutta Residual Distribution. [Research Report] RR-8367, INRIA. 2013. hal-00863154v2

\section{HAL Id: hal-00863154 \\ https://hal.inria.fr/hal-00863154v2}

Submitted on 18 Sep 2013

HAL is a multi-disciplinary open access archive for the deposit and dissemination of scientific research documents, whether they are published or not. The documents may come from teaching and research institutions in France or abroad, or from public or private research centers.
L'archive ouverte pluridisciplinaire HAL, est destinée au dépôt et à la diffusion de documents scientifiques de niveau recherche, publiés ou non, émanant des établissements d'enseignement et de recherche français ou étrangers, des laboratoires publics ou privés. 


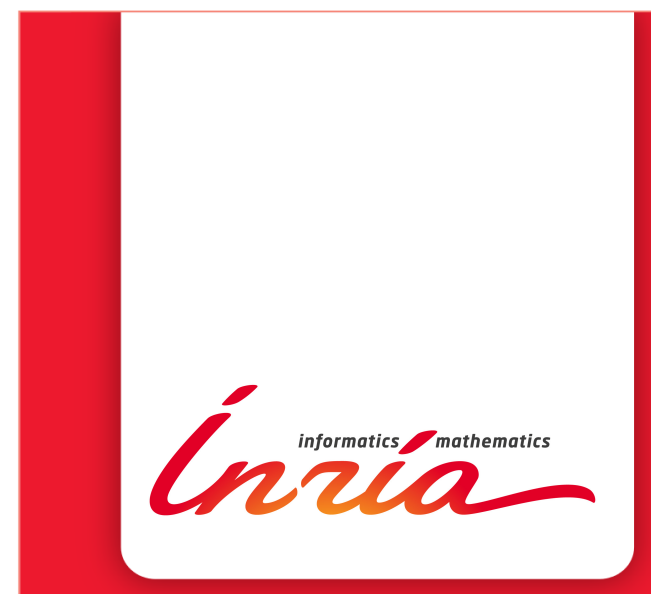

An ALE formulation for explicit Runge-Kutta Residual Distribution

R. Abgrall, L. Arpaia, M. Ricchiuto

RESEARCH

REPORT

$\mathrm{N}^{\circ} 8367$

July 2013

Project-Teams BACCHUS 



\title{
Inĩáa
}

\section{An ALE formulation for explicit Runge-Kutta} Residual Distribution

\author{
R. Abgrall: L. Arpaia*, M. Ricchiuto* \\ Project-Teams BACCHUS \\ Research Report n 8367 - July 2013 - 47 pages
}

\begin{abstract}
In this paper we consider the solution of hyperbolic conservation laws on moving meshes by means of an Arbitrary Lagrangian Eulerian (ALE) formulation. In particular we propose an ALE framework for the genuinely explicit residual distribution schemes of (Ricchiuto and Abgrall J.Comput.Phys 229, 2010). The discretizations obtained are thoroughly tested on a large number of benchmarks
\end{abstract}

Key-words: Hyperbolic conservation laws, moving grids, ALE formalism, unstructured grids, residual distribution

* Inria Bordeaux - Sud-Ouest, Team BACCHUS

\section{RESEARCH CENTRE \\ BORDEAUX - SUD-OUEST}

351, Cours de la Libération

Bâtiment A 29

33405 Talence Cedex 


\section{An ALE formulation for explicit Runge-Kutta Residual Distribution}

Résumé : Dans ce travail on considère la resolution de lois de conservation sur maillages mobiles par une formulation Arbitrary Lagrangian Eulerian (ALE). On propose en particulier un formalisme ALE pour les schémas RD explicites de (Ricchiuto and Abgrall J.Comput.Phys 229, 2010). Les schémas ainsi obtenus sont testés sur des nombreux benchmarks.

Mots-clés : Hyperbolic conservation laws, moving grids, ALE formalism, unstructured grids, residual distribution 


\section{Contents}

\begin{tabular}{lll}
\hline & Introduction & 4
\end{tabular}

\begin{tabular}{|ll|l}
2 & Scalar Conservation laws in Arbitrary Lagrangian Eulerian formulation & 5
\end{tabular}

$\begin{array}{lll}3 & \text { Residual Distribution for 2D scalar conservation laws } & 8\end{array}$

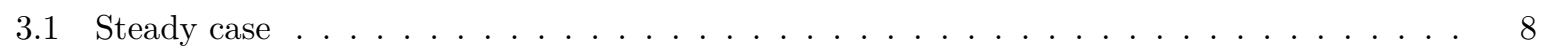

3.2 Extension to time dependent problems $\ldots \ldots \ldots \ldots \ldots \ldots \ldots$

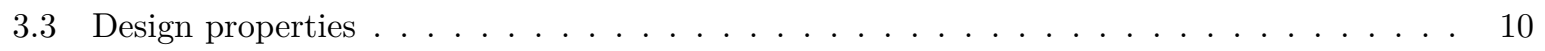

$3.3 .1 \quad$ Nonlinear conservation laws and conservation . . . . . . . . . . . . . . . . . . 10

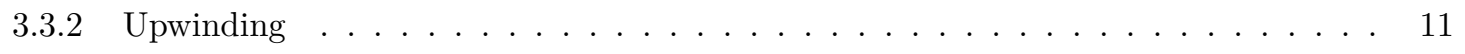

$3.3 .3 \quad$ Positivity and discrete maximum principle $\ldots \ldots \ldots \ldots \ldots \ldots \ldots$. . . . . . . . . 12

$3.3 .4 \quad$ Order of accuracy and Godunov Theorem . . . . . . . . . . . . . . . . . 12

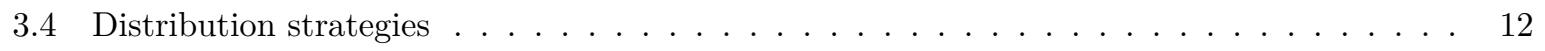

$3.4 .1 \quad$ Linear positive schemes $\ldots \ldots \ldots \ldots \ldots \ldots$. . . . . . . . . . . . . . 12

3.4 .2 Linear second order schemes $\ldots \ldots \ldots \ldots \ldots$

3.4 .3 Nonlinear schemes $\ldots \ldots \ldots \ldots \ldots \ldots$

$3.5 \quad$ Genuinely explicit RK-RD time marching procedure $\ldots \ldots \ldots \ldots \ldots \ldots$

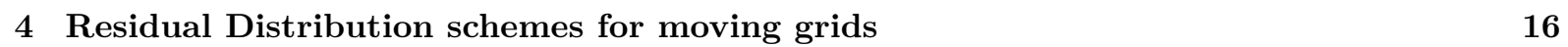

4.1 Galerkin Finite Element method $\ldots \ldots \ldots \ldots \ldots \ldots$

4.2 An example of a DGCL satisfying scheme $\ldots \ldots \ldots \ldots \ldots \ldots \ldots \ldots$

4.3 An approach "à la Farhat" $\ldots \ldots \ldots \ldots \ldots$

$4.3 .1 \quad$ Explicit Euler . . . . . . . . . . . . . . . . . . . . . . . . . . . . . 19

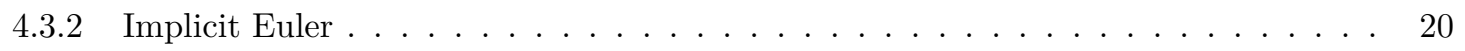

4.3 .3 Crank-Nicholson . . . . . . . . . . . . . . . . . . . . . . 20

$4.4 \quad$ Stabilized Finite Elements and Residual Distribution . . . . . . . . . . . . . . . . . . . . . 20

4.4 .1 Dobes Closure for explicit Euler time stepping . . . . . . . . . . . . . . . . . 21

$4.4 .2 \quad$ Another closure for explicit Euler time stepping . . . . . . . . . . . . . . . . 21

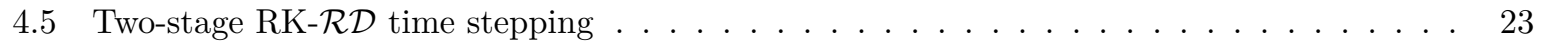

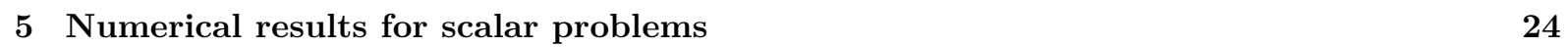

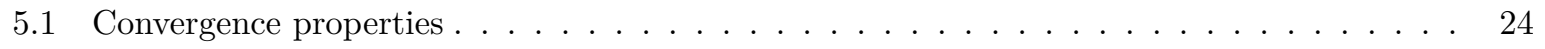

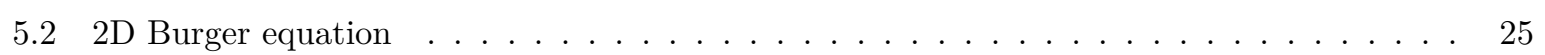


$6 \quad$ Application to the perfect gas Euler Equations $\quad 30$

6.1 Implementation details . . . . . . . . . . . . . . . . . . . . . . . . . 30

6.2 Numerical results . . . . . . . . . . . . . . . . . . . . . . . . . . . . . . . . 31

6.2 .1 Advection of a Vortex . . . . . . . . . . . . . . . . . . . . 32

$6.2 .2 \quad$ A 2D Riemann problem . . . . . . . . . . . . . . . . . . . 34

$6.2 .3 \quad$ An application: wind tunnel with wall deflection . . . . . . . . . . . . . . . . 39

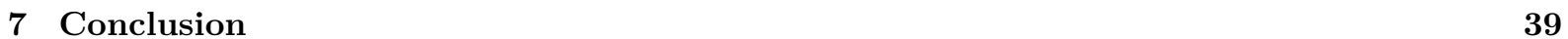

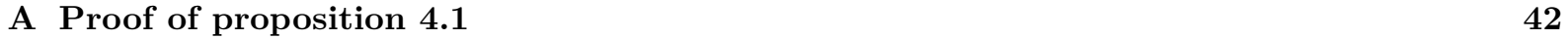

\section{Introduction}

Many unsteady problems governed by conservation laws involve the movement of the boundaries. In the numerical approximation of this phenomena additional difficulties arise because the grid must adapt at every time step to the moving boundaries. If equations are written in an Eulerian framework, this makes necessary an intermediate step between the computation of two successive numerical solutions. In fact, once the grid has been adapted to the new boundaries, an interpolation of the previous solution over the new grid is essential in order to start up the computation of the new solution. On the opposite conservation laws can be written in a Lagrangian framework, the grid is moved at the flow velocity and the integral conservation laws are written always for the same particles. The algorithm works always on the same grid, with the same nodes, and no interpolation step is needed. The problem of this approach is that the grid movement is connected to the particles paths and when strong distortion are present, like in a fluid dynamic context, the method suffer from instabilities because of mesh tangling: a conservative remap step is needed, see e.g. [?] among many other.

The Arbitrary Lagrangian Eulerian formulation is another way of dealing with mesh movement and solves the drawbacks of both the approaches since conservation laws are written for an arbitrary moving grid with respect to the particles motion. In the case of large deformation, modifications of the mesh become mandatory, as in the Lagrangian methods. An elegant way of dealing with ALE and large deformations is described in [?] and this method can be coupled with mesh refinement.

The appearance of the ALE approach dates back to the early eighties due to the contribution of J.Donea [1. The idea was found very appealing in many field of computational continuum mechanics because the extension of a classical Lagrangian (for solid mechanics) or Eulerian (for fluid mechanics) method into ALE formulation is straightforward and requires few lines of changes in the algorithm. In fluid dynamics the recasting of Eulerian Finite Volumes and Finite Elements has been investigated since long time, see e.g. [?, ?, 2].

In this work, we have foccussed on the formulation of the ALE approach within a Residual Distribution method. Residual Distribution $(\mathcal{R D})$ schemes represent nowadays an alternative to both Finite Volume $(\mathrm{FV})$ and Finite Elements $(\mathrm{FE})$. The road to $\mathcal{R} \mathcal{D}$ was paved by the early work of Ni, Rice and Schnipke and T.J. Hughes on residual based schemes for hyperbolic problems [3, 4, 5], and finally by the fluctuation splitting approach of P.L. Roe and co-workers [6, 7]. Many of the subsequent developments are due to the group of H. Deconinck at the von Karman Institute for Fluid dynamics. In particular, ALE formulations of $\mathcal{R D}$ have been proposed in the work of Michler and Deconinck [8], who achieved first order with an 
Explicit Euler time integrator, and later Dobes and Deconinck (see e.g. [9]) who moved to high order time approximation (BDF3, Crank Nicholson), this obtaining have second order of accuracy.

The aim of this work is to obtain a numerical solution with second order of accuracy using a faster explicit Runge Kutta time integrator. This is achieved using the genuinely explicit formulation proposed in [10], and combining it with a ALE formalism. The paper is organized as follows. First we recall the scalar conservation laws in Arbitrary Lagrangian Eulerian form, then a brief summary on scalar Residual Distribution schemes on fixed grids is given. In section $\S 3$, always working on fixed grids, we recall the RD-RK time marching procedure of [10]. The scheme is extended to conservation laws in ALE form in section $\S 4$. In section $\S 5$ two scalar test cases are used to verify the scheme's positivity and convergence. Finally the scalar algorithm is extended to systems of conservation laws. Section $\S 6$ is devoted to Euler Equations of gasdynamics.

\section{Scalar Conservation laws in Arbitrary Lagrangian Eulerian formulation}

We assume that we are given a domain $\Omega$ and a field of displacements that brings every point of the domain from the reference position $\boldsymbol{X}$ to the actual one $\boldsymbol{x}(t)$ and that this field is governed by an arbitrary given motion law

$$
\frac{d \boldsymbol{x}(t)}{d t}=\boldsymbol{\sigma}(\boldsymbol{x}, t)
$$

Solving the ODE (1) starting from the reference configuration the actual configuration through the following mapping is

$$
A(t): \Omega_{X} \rightarrow \Omega_{x}(t), \quad \boldsymbol{x}=A(\boldsymbol{X}, t)
$$

with the condition $A(\boldsymbol{X}, 0)=\boldsymbol{X}$. We define the Jacobian matrix of the mapping as

$$
\mathcal{J}_{A}=\frac{\partial \boldsymbol{x}}{\partial \boldsymbol{X}}
$$

and assume that $J_{A}=\operatorname{det} \mathcal{J}_{A} \neq 0$, i.e. the mapping $A$ is assumed to be invertible. We introduce now another set of coordinates, the Lagrangian or material coordinates $\chi$, and a mapping that describes the motion of each particle. This mapping returns the physical location, represented by the actual coordinate $\boldsymbol{x}$, of the particle marked with $\chi$ at time $t$

$$
B(t): \Omega_{\chi} \rightarrow \Omega_{x}(t), \quad \boldsymbol{x}=B(\chi, t) \text { with } B(\chi, 0)=\chi
$$

Again, the Jacobian matrix of the mapping $\mathcal{J}_{B}=\frac{\partial \boldsymbol{x}}{\partial \boldsymbol{X}}$ is assumed to satisfy $J_{B}=\operatorname{det} \mathcal{J}_{B} \neq 0$, i.e. the mapping $B$ is invertible.

If $u$ is a conserved quantity it can be expressed as a function of the different coordinates $\boldsymbol{x}, \boldsymbol{X}, \boldsymbol{\chi}$ and three different time derivatives can be defined. If the derivation is computed in the actual configuration, we define the spatial derivative:

$$
\left.\frac{\partial u(\boldsymbol{x}, t)}{\partial t}\right|_{x}=\frac{\partial u}{\partial t} .
$$

If it is computed following the particle motion one has the material derivative:

$$
\left.\frac{\partial u(\boldsymbol{\chi}, t)}{\partial t}\right|_{\chi}=\frac{d u}{d t} .
$$


Finally if it is computed following the domain motion one has the referential derivative:

$$
\left.\frac{\partial u(\boldsymbol{X}, t)}{\partial t}\right|_{X}
$$

Moreover two different velocities can be computed: the particle velocity and the domain velocity

$$
\begin{gathered}
\left.\frac{\partial x(t)}{\partial t}\right|_{X}=\frac{d A(\boldsymbol{X}, t)}{d t}=\boldsymbol{\sigma} \\
\left.\frac{\partial x(t)}{\partial t}\right|_{\chi}=\frac{d B(\boldsymbol{\chi}, t)}{d t}=\boldsymbol{a}
\end{gathered}
$$

The chain rule provides a relation between the above derivatives and these velocities

$$
\begin{gathered}
\frac{d u}{d t}=\frac{\partial u}{\partial t}+\boldsymbol{a}(\boldsymbol{x}, t) \cdot \nabla u(\boldsymbol{x}, t) \\
\frac{d u}{d t}=\left.\frac{\partial u}{\partial t}\right|_{X}+(\boldsymbol{a}(\boldsymbol{x}, t)-\boldsymbol{\sigma}(\boldsymbol{x}, t)) \cdot \nabla u(\boldsymbol{x}, t)
\end{gathered}
$$

From continuum mechanics we also have the following

$$
\begin{gathered}
\left.\frac{\partial J_{B}}{\partial t}\right|_{\chi}=\frac{d J_{B}}{d t}=J_{B} \nabla \cdot \boldsymbol{a} \\
\left.\frac{\partial J_{A}}{\partial t}\right|_{X}=J_{A} \nabla \cdot \boldsymbol{\sigma} .
\end{gathered}
$$

This last relation is commonly called Geometric Conservation Law (GCL) and represents a constraint the points of the domain have to satisfy during their arbitrary motion. This relation plays a very important role when developing a numerical method with a moving grid; up to now we only want to make clear that the movement of the domain is arbitrary but within hypothesis 12 .

The conservation of the scalar quantity $u$ can be written, depending on convenience, in the different coordinate frameworks. If we choose a material control volume $C(t)$ which contains always the same particles, following them throughout all the domain, the conservation is simply stated in actual coordinates

$$
\frac{d}{d t} \int_{C(t)} u(\boldsymbol{x}, t) d \boldsymbol{x}=0
$$

Passing to material coordinates and using (11) together with the chain rule (9)

$$
\begin{aligned}
\frac{d}{d t} \int_{C_{\chi}} u(\boldsymbol{X}, t) J_{B} d \boldsymbol{X} & =\int_{C_{\chi}} \frac{d}{d t}\left(u(\chi, t) J_{B}\right) d \boldsymbol{\chi} \\
& =\int_{C_{\chi}}\left(\frac{d u}{d t} J_{B}+u \frac{d J_{B}}{d t}\right) d \boldsymbol{\chi} \\
& =\int_{C_{\chi}}\left(\frac{\partial u}{\partial t}+\boldsymbol{a} \cdot \nabla u+u \nabla \cdot \boldsymbol{a}\right) J_{B} d \boldsymbol{\chi}
\end{aligned}
$$

We have derived the conservation law in integral Eulerian form

$$
\int_{C}\left(\frac{\partial u}{\partial t}+\nabla \cdot \boldsymbol{f}\right) d \boldsymbol{x}=0
$$


Now, in (14), we use (10) instead of (9)

$$
\begin{aligned}
\int_{C_{\chi}}\left(\frac{d u}{d t} J_{B}+u \frac{d J_{B}}{d t}\right) d \boldsymbol{\chi} & =\int_{C_{\chi}}\left(\left.\frac{\partial u}{\partial t}\right|_{X}+(\boldsymbol{a}-\boldsymbol{\sigma}) \cdot \nabla u+u \nabla \cdot \boldsymbol{a}\right) J_{B} d \boldsymbol{\chi} \\
& =\int_{C(t)}\left(\left.\frac{\partial u}{\partial t}\right|_{X}+\nabla \cdot \boldsymbol{f}-\boldsymbol{\sigma} \cdot \nabla u\right) d \boldsymbol{x}
\end{aligned}
$$

The first term can be rewritten if we compute the derivative of the conserved quantity inside a control volume $C(t)$, which is following the motion of the points of the domain. Note that there is a little abuse in the notation since $C(t)$ has been already used to represent a material volume. Transforming into referential coordinate and using the fact that $C_{X}$ does not depend on time

$$
\begin{aligned}
\left.\frac{\partial}{\partial t}\right|_{X} \int_{C(t)} u(\boldsymbol{x}, t) d \boldsymbol{x} & =\left.\frac{\partial}{\partial t}\right|_{X} \int_{C_{X}} u(\boldsymbol{X}, t) J_{A} d \boldsymbol{X} \\
& =\left.\int_{C_{X}} \frac{\partial u}{\partial t}\right|_{X} J_{A} d \boldsymbol{X}+\left.\int_{C_{X}} \frac{\partial J_{A}}{\partial t}\right|_{X} u d \boldsymbol{X} \\
& =\left.\int_{C_{X}} \frac{\partial u}{\partial t}\right|_{X} J_{A} d \boldsymbol{X}+\int_{C_{X}} J_{A} u \nabla \cdot \boldsymbol{\sigma} d \boldsymbol{X}
\end{aligned}
$$

So we have

$$
\left.\int_{C(t)} \frac{\partial u}{\partial t}\right|_{X} d \boldsymbol{x}=\left.\frac{\partial}{\partial t}\right|_{X} \int_{C(t)} u d \boldsymbol{x}-\int_{C(t)} u \nabla \cdot \boldsymbol{\sigma} d \boldsymbol{x}
$$

Substituting 18 in $(16)$ we get the integral form of conservation law written in Arbitrary Lagrangian Eulerian Formulation (ALE)

$$
\left.\frac{\partial}{\partial t}\right|_{X} \int_{C(t)} u d \boldsymbol{x}+\int_{C(t)} \nabla \cdot(\boldsymbol{f}-u \boldsymbol{\sigma}) d \boldsymbol{x}=0
$$

which express the conservation of $u$ contained in a moving arbitrary control volume. The equilibrium is reached by the relative flux of $u$ entering and leaving the volume with velocity $\boldsymbol{a}-\boldsymbol{\sigma}$.

It is interesting to note that the ALE formulation rapresents a generalization of both the Eulerian and Lagrangian formulations. In fact in (19)

1. If $\boldsymbol{\sigma}=\mathbf{0}$, the control volume is fixed in space (from $C(t)$ to $C$ ) and we get the Eulerian form 15 ,

2. If $\boldsymbol{\sigma}=\boldsymbol{a}$, the control volume is moving with the particle motion and we get the Lagrangian form (13)

A differential form of conservation law in ALE formulation is needed but its derivation is simple if we start from the integral form 19 and we use 12

$$
\begin{aligned}
& \left.\frac{\partial}{\partial t}\right|_{X} \int_{C_{X}} u J_{A} d \boldsymbol{X}+\int_{C_{X}} J_{A} \nabla \cdot(\boldsymbol{f}-u \boldsymbol{\sigma}) d \boldsymbol{X}= \\
& =\int_{C_{X}}\left(\left.\frac{\partial\left(J_{A} u\right)}{\partial t}\right|_{X}+J_{A} \nabla \cdot(\boldsymbol{f}-u \boldsymbol{\sigma})\right) d \boldsymbol{X}=0
\end{aligned}
$$

Using the localization principle, the differential form of conservation law in ALE formulation is derived

$$
\left.\frac{\partial\left(J_{A} u\right)}{\partial t}\right|_{X}+J_{A} \nabla \cdot(\boldsymbol{f}-u \boldsymbol{\sigma})=0
$$


It is easy to see that the requirement for volume conservation (12) we have previously done can be derived simply by imposing a state of uniform flow in (20). In this case we are modelling a situation in which the flow is uniform and the domain is moving from behind.

Developing the derivative in 20 and then substituting 12

$$
\left.J_{A} \frac{\partial u}{\partial t}\right|_{X}+J_{A} u \nabla \cdot \boldsymbol{\sigma}+J_{A} \nabla \cdot(\boldsymbol{f}-u \boldsymbol{\sigma})=0
$$

which lead to the following equation that we will use extensively hereinafter

$$
\left.\frac{\partial u}{\partial t}\right|_{X}+\nabla \cdot \boldsymbol{f}-\boldsymbol{\sigma} \cdot \nabla u=0
$$

\section{Residual Distribution for $2 \mathrm{D}$ scalar conservation laws}

In this section, we consider the numerical approximation of the hyperbolic conservation law derived from 15

$$
\frac{\partial u}{\partial t}+\nabla \cdot \boldsymbol{f}(u)=0 \quad \text { in } \Omega \in \mathbb{R}^{2}, t \in[0, T]
$$

We recall how the Residual Distribution discrete second order approximation of 22 is obtained. For simplicity, we restrict ourselves to the two dimensional case, but all the discussion and the sub-sequent developments carry on to three space dimensions without any modification. We start by presenting the basic prototype scheme for steady problems, then recall one possible extension to time dependent computations. We also recall the main design properties for these schemes.

\subsection{Steady case}

Consider the steady scalar conservation law

$$
\nabla \cdot \boldsymbol{f}(u)=0, \quad \boldsymbol{x} \in \Omega
$$

We have approximated the domain through a suitable triangulation $\mathcal{T}_{h}$. The letter $K$ denotes a generic triangle, and the list of vertices of $\mathcal{T}_{h}$ is $\left\{M_{i}\right\}_{i=1, n_{v}}$. By abuse of language, we identify the vertex $M_{j}$ and its index $j$. We consider a globaly continuous approximation which is piecewice linear approximation over each triangle

$$
u^{h}(\boldsymbol{x}, t)=\sum_{j=1}^{n_{v}} \varphi_{j}(\boldsymbol{x}) u_{j}(t)
$$

with $\varphi_{j}$ the standard continuous piecewise linear Lagrange basis functions. The Residual Distribution approximation is then obtained as follows (boundary conditions are neglected)

1. On each element $K$ compute the residual

$$
\phi^{K}=\int_{K} \nabla \cdot \boldsymbol{f}\left(u^{h}\right) d \boldsymbol{x}=\int_{\partial K} \boldsymbol{f}\left(u^{h}\right) \cdot \boldsymbol{n} d s
$$

2. Distribute the residuals to the nodes of the element $i, j, k \in K$

$$
\phi^{K}=\sum_{j \in K} \phi_{j}^{K}
$$


3. Assemble elemental contributions. If $\mathcal{D}_{i}$ is the set of all the elements sharing node $i$, we have

$$
\sum_{K \in \mathcal{D}_{i}} \phi_{i}^{K}=0, \quad \forall i \in \mathcal{T}_{h}
$$

Of course, the main step is the decomposition (24). We need to design the sub-residuals $\phi_{j}^{K}$ in such a way that stability and convergence is garantied. The conservation relation (24) can be shown, adding the same assumptions as in the Lax Wendrof theorem, that the limit solution, if it exist, is a weak solution of $[23$, , see [?].

In practice, and in this simplified setting, we can also write the subresiduals $\phi_{i}^{K}$ as fractions of the total residual $\phi^{K}$,

$$
\phi_{i}^{K}=\beta_{i}^{K} \phi^{K}
$$

and the conservation relation is rephrased as $\sum_{i \in K} \beta_{i}^{K}=1$. The properties of the $\mathcal{R D}$ scheme are translated into properties of the $\beta_{i}^{K}$, we come to that point latter in the text.

\subsection{Extension to time dependent problems}

Consider now the time dependent advection equation ${ }^{1}$

$$
L(u)=\frac{\partial u}{\partial t}+\boldsymbol{a} \cdot \nabla u=0 \quad \text { in } \Omega \in \mathbb{R}^{2}, t \in[0, T]
$$

The extension of the schemes presented in the previous paragraph is done using the analogy with stabilized FE schemes introduced in [11, 12, 13. (see also [14, 10]). The discrete counterpart of (26) is written as

$$
\sum_{K \in \mathcal{T}_{h}} \sum_{j \in K} \int_{K} w_{i} \varphi_{j} \frac{d u_{j}}{d t} d \boldsymbol{x}+\sum_{K \in \mathcal{T}_{h}} \int_{K} w_{i} \boldsymbol{a} \cdot \nabla u_{h} d \boldsymbol{x}=0 \quad \forall i \in \mathcal{T}_{h}
$$

with a test function $w_{i}=\varphi_{i}+\gamma_{i}$ which satisfies

$$
\frac{1}{|K|} \int_{K} w_{i} d \boldsymbol{x}=\beta_{i}^{K}
$$

Introducing the mass-matrix $m_{i j}^{K}=\int_{K} \varphi_{j} w_{i} d \boldsymbol{x}$, using the fact that $\left.\nabla u_{h}\right|_{K}$ is constant in the $\mathbb{P}^{1}$ case and condition (28), we obtain the time dependent generalization of $\mathcal{R D}$ scheme:

$$
\sum_{K \in \mathcal{D}_{i}} \sum_{j \in K} m_{i j}^{K} \frac{d u_{j}}{d t}+\sum_{K \in \mathcal{D}_{i}} \beta_{i}^{K} \phi^{K}=0
$$

Several possible definition of $w_{i}$ allow to recover a given $\beta_{i}^{K}$, a discussion of this issue can be found in [10], and is beyond the scope of this paper. Note that the only place where we really use the fact that (i) the elements are triangles, (ii) we use linear approximation and (iii) the velocity field is constant is to go from (27) to 29 .

Here we recall two possible formulations, called respectively F1 and F2 [10]:

$$
\left.w_{i}^{F 1}\right|_{K}=\beta_{i}^{K} \quad \text { and }\left.\quad w_{i}^{F 2}\right|_{K}=\varphi_{i}(\boldsymbol{x})+\beta_{i}^{K}-\frac{1}{3}
$$

\footnotetext{
${ }^{1}$ we assume $\boldsymbol{a}$ to be constant, however see section $\S 2.3 .1$
} 


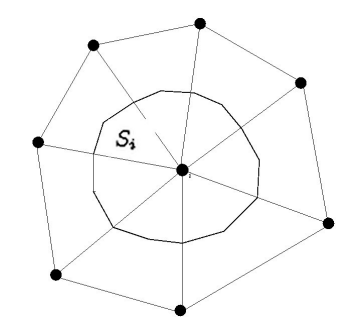

Figure 1: Median Duall Cell $S_{i}$

The corresponding expressions for the mass matrices $\operatorname{ar} e^{2}$

$$
m_{i j}^{F 1}=\frac{|K|}{3} \beta_{i}^{K}=|K| \hat{m}_{i j}^{F 1} \quad \text { and } \quad m_{i j}^{F 2}=\frac{|K|}{3}\left(\frac{\delta_{i j}}{4}+\beta_{i}^{K}-\frac{1}{12}\right)=|K| \hat{m}_{i j}^{F 2}
$$

For both formulations, (row-wise) mass lumping leads to

$$
m_{i j}^{M L}=\frac{|K|}{3} \delta_{i j}
$$

Defining the median dual cell $\left|S_{i}\right|=\sum_{K \in \mathcal{D}_{i}} \frac{|K|}{3}$ (cf figure 1), we obtain the Mass Lumped (ML) formulation of $\mathcal{R D}$

$$
\left|S_{i}\right| \frac{d u_{i}}{d t}+\sum_{K \in \mathcal{D}_{i}} \phi_{i}^{K}=0
$$

\subsection{Design properties}

\subsubsection{Nonlinear conservation laws and conservation}

The link between (26) and the more general case of $(22)$ is hidden in the computation of the element residuals $\phi_{j}^{K}$. Very simple algebraic manipulations show that

$$
\begin{aligned}
\sum_{i \in \mathcal{T}_{h}}\left(\sum_{K \in \mathcal{D}_{i}} \sum_{j \in K} m_{i j}^{K} \frac{d u_{j}}{d t}+\sum_{K \in \mathcal{D}_{i}} \phi_{i}^{K}\right) & =\sum_{K \in \mathcal{T}_{h}}\left(\sum_{i, j \in K} m_{i j}^{K} \frac{d u_{j}}{d t}+\sum_{j \in K} \phi_{j}^{K}\right) \\
& =\int_{\Omega_{h}} \frac{d u_{h}}{d t} d \boldsymbol{x}+\sum_{K \in \mathcal{T}_{h}} \phi^{K}
\end{aligned}
$$

so that a sufficient condition to recover a global conservation statement is that

$$
\phi^{K}=\int_{\partial K} \boldsymbol{f}\left(u^{h}\right) \cdot \boldsymbol{n} d s
$$

For a more sound mathematical justification of the last condition, including a Lax-Wendroff theorem, the reader can refer to e.g. [15] and references therein. Note that in practice (34) can be satisfied either

\footnotetext{
${ }^{2}$ the superscript ${ }^{K}$ is omitted to simplify notation
} 
by directly using contour integration to compute $\phi^{K}$, as suggested in [16, 17, or by introducing an exact Jacobian mean value linearization :

$$
\overline{\boldsymbol{a}}=\frac{1}{|K|} \int_{K} \boldsymbol{a}\left(u^{h}\right) d \boldsymbol{x}=\frac{1}{|K|} \int_{K} \frac{\partial \boldsymbol{f}\left(u^{h}\right)}{\partial u} d \boldsymbol{x}
$$

so that in the $\mathbb{P}^{1}$ case, using the properties of the basis functions, we can write

$$
\begin{aligned}
\phi^{K}=\int_{\partial K} \boldsymbol{f}\left(u^{h}\right) \cdot \boldsymbol{n} d s & =\int_{K} \nabla \cdot \boldsymbol{f}\left(u^{h}\right) d \boldsymbol{x}=\int_{K} \boldsymbol{a}\left(u^{h}\right) \cdot \nabla u^{h} d \boldsymbol{x} \\
& =\overline{\boldsymbol{a}} \cdot \nabla u^{h}|K|=\frac{1}{2} \sum_{j \in K} \overline{\boldsymbol{a}} \cdot \boldsymbol{n}_{j} u_{j}=\sum_{j \in K} k_{j} u_{j},
\end{aligned}
$$

having introduced the upwind parameter

$$
k_{i}=\frac{1}{2} \overline{\boldsymbol{a}} \cdot \boldsymbol{n}_{i}
$$

with $\boldsymbol{n}_{i}$ the inward normal to the edge facing node $i$, scaled by the edge length. Since $\sum_{j \in K} k_{j}=0$ we also have

$$
\phi^{K}=\sum_{j \in K, j \neq i} k_{j}\left(u_{j}-u_{i}\right)
$$

Whether a conservative linearization is used in practice or not, in the following we will make use of (35), unduly assuming the equivalence between the fully nonlinear problem and the locally linearize done, i.e.

$$
k_{i}=\int_{K} \boldsymbol{a}\left(u^{h}\right) \cdot \nabla \varphi_{i} d \boldsymbol{x} .
$$

In particular, this allows to recast any $\mathcal{R D}$ scheme as

$$
\sum_{K \in \mathcal{D}_{i}} \sum_{j \in K} m_{i j}^{K} \frac{d u_{j}}{d t}=-\sum_{K \in \mathcal{D}_{i}} \sum_{j \in K, j \neq i} c_{i j}^{K}\left(u_{i}-u_{j}\right) \quad \forall i \in \mathcal{T}_{h}
$$

and, in the mass-lumped case, as

$$
\left|S_{i}\right| \frac{d u_{i}}{d t}=-\sum_{K \in \mathcal{D}_{i}} \sum_{j \in K, j \neq i} c_{i j}^{K}\left(u_{i}-u_{j}\right) \quad \forall i \in \mathcal{T}_{h}
$$

\subsubsection{Upwinding}

The upwind parameters $k_{i}$ (cf. equation (35) ) allow to distinguish between upstream nodes and downstream nodes in a given element. In particular, if $k_{i}>0$ node $i$ is downstream, while $k_{i}<0$ for upstream nodes. Multidimensional upwinding, as introduced by Roe, Deconinck and collaborators (see e.g. [18]), corresponds to the condition

$$
k_{i}<0 \Rightarrow \beta_{i}=0
$$

Multidimensional upwind schemes have been shown to have much reduced numerical dissipation compared to classical upwind finite volume schemes [19, 20]. 


\subsubsection{Positivity and discrete maximum principle}

The theory of positive coefficient schemes 21 is the guideline underpinning the construction of nonoscillatory $\mathcal{R D}$ schemes. It is classically formulated for the simplified prototype (39). Here we will say that a scheme is positive, if in (39) we have $c_{i j}^{K} \geq 0, \forall i, j \in K$ and for all element $K$. In this sense, positivity can be shown to be equivalent to the so-called Local Extremum Diminishing property [22, 23, 24, and, provided that (39) is integrated with a SSP time marching scheme it leads to a discrete maximum principle, under a time step restriction. For example, when the explicit Euler scheme is used, one readily shows that

$$
\left|S_{i}\right| \frac{u_{i}^{n+1}-u_{i}^{n}}{\Delta t}=-\sum_{K \in \mathcal{D}_{i}} \sum_{j \neq i} c_{i j}^{K} u_{i}^{n}-u_{j}^{n} \text { implies } \min _{j \in \mathcal{D}_{i}} u_{j}^{n} \leq_{i}^{n+1} \leq \max _{j \in \mathcal{D}_{i}} u_{j}^{n}
$$

provided that $c_{i j}^{K} \geq 0$ and $\Delta t \sum_{K \in \mathcal{D}_{i}} \sum_{j \neq i} c_{i j}^{K} \leq\left|S_{i}\right|$.

For more details the interested reader can refer to e.g. [14, 24] and references therein.

\subsubsection{Order of accuracy and Godunov Theorem}

Detailed analysis of the accuracy of $\mathcal{R D}$ schemes, and the related constraints on the discretization can be found in [19, 25] for the steady case, and in [26, 24, 10, for the time dependent case. In the $\mathbb{P}^{1}$ case, the main result is that schemes admitting a set of uniformly bounded distribution coefficients are second order accurate.

As shown e.g. in [19], a generalization to $\mathcal{R D}$ of Godunov's theorem [27, states that a linear scheme cannot be positive and second order simultaneously. Some nonlinearity is necessary to combine both properties. This point is addressed in section 3.4 .2

\subsection{Distribution strategies}

We briefly discuss the distribution strategies later tested in the numerical benchmarks. Then, we present first examples of linear schemes, which are either positive or second order accurate. Last, we describe how nonlinear schemes are obtained.

\subsubsection{Linear positive schemes}

In this paper we consider two linear positive schemes. The first is a $\mathcal{R D}$ formulation of the Lax-Friedrich's scheme for which the steady part of the discrete equations is obtained by setting

$$
\phi_{i}^{K}=\phi_{i}^{L x F}=\frac{1}{3} \phi^{K}+\frac{\alpha^{K}}{3} \sum_{\substack{j \in K \\ j \neq i}}\left(u_{i}-u_{j}\right)
$$

The scheme can be shown to be positive under the condition $\alpha^{K} \geq \max _{j \in K}\left|k_{j}\right|$.

The second linear positive scheme we use is Roe's optimal $\mathrm{N}$ scheme [7, obtained by setting

$$
\phi_{i}^{K}=\phi_{i}^{N}=k_{i}^{+}\left(u_{i}-u_{i n}\right)
$$


where the inflow state is computed

$$
u_{i n}=-\sum_{j \in K} N k_{j}^{-} u_{j}, \text { with } N=\left(\sum_{j \in K} k_{j}^{+}\right)^{-1}
$$

The scheme is readily shown to be positive [7] and is multidimensional upwind. In this form, in the nonlinear case the $\mathrm{N}$ scheme is conservative only if an exact Jacobian linearization is used. A modified variant, formally very similar, but allowing to compute the element residual directly by contour integration, is discussed in detail in [16, 17].

Note that for both the LxF and N schemes, the distribution coefficients are not explicitly defined, which makes the use of the Petrov-Galerkin analogy of section $\$ 1.2$ impossible. For this reason, these schemes are usually integrated in time using the lumped formulation (39).

\subsubsection{Linear second order schemes}

We will test two linear second order schemes. The first is a $\mathcal{R} \mathcal{D}$ reformulation of the SUPG scheme of [5], obtained simply by setting

$$
\beta_{i}^{K}=\beta_{i}^{S U P G}=\frac{1}{3}+\frac{1}{|K|} k_{i} \tau
$$

In our tests we have set $\tau=|K|\left(\sum_{j \in K}\left|k_{j}\right|\right)^{-1} / 2$.

The second scheme we have tested is the multidimensional upwind LDA scheme [28] obtained by setting

$$
\beta_{i}^{K}=\beta_{i}^{L D A}=k_{i}^{+} N
$$

\subsubsection{Nonlinear schemes}

In this paper we compare results obtained with two different strategies to construct nonlinear discretizations. The first, is based on a blending of a high order and of a positive linear schemes. In the steady case, the B scheme is defined by

$$
\phi_{i}^{K}=\phi_{i}^{B}=\left(1-l\left(u^{h}\right)\right) \phi_{i}^{\mathcal{H O}}+l\left(u^{h}\right) \phi_{i}^{\mathcal{P}}
$$

where the blending coefficient $l\left(u^{h}\right)$ has to be of order $\mathcal{O}(h)$ (or smaller) when the solution is smooth and of order $l\left(u^{h}\right) \approx 1$ when the solution is discontinuous. Several definition of this coefficient are possible and we refer to [19] for a thorough discussion. Here, we have tested the heuristic definition proposed by Deconinck and co-workers (see e.g. in [29] and references therein)

$$
l=\frac{\left|\phi^{K}\right|}{\sum_{j}\left|\phi_{j}^{\mathcal{P}}\right|}
$$

In particular, we have tested the multidimensional upwind LDA-N scheme obtained by blending the LDA and the $\mathrm{N}$ scheme. Note that in the time dependent case, the mass matrix of the LDA-N becomes

$$
m_{i j}^{L D A-N}=\left(1-l\left(u^{h}\right)\right) m_{i j}^{L D A}+l\left(u^{h}\right) \frac{|K|}{3} \delta_{i j}
$$


while the blending parameter should now include the whole residual, namely

$$
l=\frac{\left|\Phi^{K}\right|}{\sum_{j}\left|\Phi_{j}^{N}\right|}
$$

with

$$
\Phi^{K}=\int_{K}\left(\frac{\partial u^{h}}{\partial t}+\nabla \cdot \boldsymbol{f}\left(u^{h}\right)\right) d \boldsymbol{x} \quad \text { and } \quad \Phi_{i}^{N}=\frac{|K|}{3} \frac{d u_{i}}{d t}+\phi_{i}^{N}
$$

Of course, the equations 490 - 50 are somewhat unclear since the meaning of $\frac{\partial u^{h}}{\partial t}$ needs to be made more precise to be able to evaluate the parameter $l$. This will be made more precise in section 3.5

We have compared the results of the multidimensional upwind LDA-N scheme with those obtained with a "non-upwind" discretization built starting from the LxF scheme. In the steady case, the main idea behind this construction is that, while in general the distribution coefficient obtained as the ratio

$$
\beta_{i}^{L x F}=\frac{\phi_{i}^{L x F}}{\phi^{K}}
$$

is unbounded, its sign gives a correct reference to build a positive scheme. In the unsteady case, we proceed as before by replacing $\phi^{K}$ by $\Phi^{K}$ as in $(50)$. The idea is then to apply a nonlinear limiter function to $\beta_{i}^{L x F}$ allowing to preserve the sign of the discretization coefficients, while yielding a bounded distribution. Several constructions satisfying these requirement are discussed in [30] to which we refer for details. In this work, we have considered the LLxF scheme obtained by setting

$$
\beta_{i}^{L L x F}=\frac{\left(\beta_{i}^{L x F}\right)^{+}}{\sum_{j \in K}\left(\beta_{j}^{L x F}\right)^{+}}
$$

where $(\cdot)^{+}=\max (0, \cdot)$.

Before introducing the explicit time marching procedure used in the paper, two remarks are necessary. The first is that, as thoroughly discussed in [31, when trying to approximate smooth solutions the LLxF scheme obtained in this way gives in practice a very erratic convergence to steady state, and yields solutions polluted by spurious modes. Instead, in presence of discontinuities, the LLxF scheme provides sharp and monotone results. As shown in the reference, this fact can be related two an ill-conditioning of the algebraic equations obtained, and, in more heuristic terms, to the lack of an upwinding mechanism. To correct this flaw in smooth regions, following [31, we have modified the distribution coefficient. In particular we will consider the LLxFs scheme which is obtained by setting

$$
\beta_{i}^{L L x F s}=\beta_{i}^{L L x F}+\delta\left(u^{h}\right) \frac{1}{|K|} k_{i} \tau
$$

where the second term is associated to the SUPG streamline diffusion, while the smoothness monitor $\delta$ is defined as

$$
\delta\left(u^{h}\right)=\min \left(1, \frac{1}{\frac{\left|\phi^{K}\right|}{\bar{u}\|\boldsymbol{a}\|_{K} h_{K}^{2}}+\varepsilon}\right)
$$

with $h_{K}$ the element reference size, $\bar{u}=\max _{j \in K}\left|u_{j}\right|$ and $\varepsilon=10^{-10}$. It is easy to show that the definition (53) can detect the discontinuties. Infact $\delta\left(u^{h}\right)$ is of order $\mathcal{O}(1)$ in smooth region where dissipation is needed to damp oscillations and of order $\mathcal{O}\left(h^{-1}\right)$ across discontinuities where the LLxF scheme behave nicely computing well-resolved profiles. Lastly, we remark that in the time dependent case, the computation of $\beta_{i}^{L L x F}$ (equation (51) ) and of $\delta$, should be done by using residuals which include in the residuals the time variation, as in equations 49 ) and 50 . 


\subsection{Genuinely explicit RK- $\mathcal{R} \mathcal{D}$ time marching procedure}

Due to the presence of the mass matrix, the use of the general prototype 29 leads inevitably to schemes requiring the solution of a nonlinear system of algebraic equations, even if explicit time integration techniques are used. For this reason, time dependent implementations of $\mathcal{R D}$ always feature some form of implicit time integration [12, 13, 14, 32, 33], or a fully coupled space-time formulation [17, 34, 35]. Moreover, positivity preservation always requires the satisfaction of time step constraints [36, 14, unless some form of nonlinear time (or space-time) discretization is used [37, 34, 35]. This leads to expensive methods, when compared to e.g. FV with Runge Kutta time integration.

The explicit RK- $\mathcal{R D}$ formulation of [10] provides one possible solution to this flaw, allowing genuinely explicit time marching. Starting from the general prototype (29], the scheme proposed in [10] is obtained as follows :

1. Time integration is performed by SSP an Runge-Kutta (RK) scheme. In this work we focus on the scheme obtained with the second order 2-stages method which, for the generic ODE

$$
\frac{d u}{d t}+e(u)=0
$$

reads

$$
\begin{array}{ll}
\frac{\Delta u_{1}}{\Delta t}+e_{1}=0, & e_{1}=e\left(u^{n}\right) \\
\frac{\Delta u_{2}}{\Delta t}+e_{2}=0, & e_{2}=\frac{1}{2}\left(e\left(u^{n}\right)+e\left(u_{1}\right)\right)
\end{array}
$$

with $\Delta u_{k}=u_{k}-u^{n}$, and $u^{n+1}=u_{2}$ and $e(u)=\nabla \cdot \boldsymbol{f}(u)$. In the following we use the notations

$$
f_{1}(u)=\nabla \cdot \boldsymbol{f}\left(u^{n}\right) \text { and } f_{2}(u)=\nabla\left(\frac{1}{2}\left(f\left(u^{n}\right)+f\left(u_{1}\right)\right) .\right.
$$

2. Each RK step is discretized in space by means of the Petrov-Galerkin statement (27), however two different approximations of the equation are used in the Galerkin part and in the bubble part, namely (cf. equation (27) and subsequent text)

$$
\int_{\Omega} \varphi_{i}\left(\frac{\Delta u_{k}^{h}}{\Delta t}+\nabla \cdot \boldsymbol{f}_{k}\left(u^{h}\right)\right) d \boldsymbol{x}+\sum_{K \in \mathcal{D}_{i}} \int_{K} \gamma_{i}\left(\frac{\Delta{\overline{u_{k}}}^{h}}{\Delta t}+\nabla \cdot \boldsymbol{f}_{k}\left(u^{h}\right)\right) d \boldsymbol{x}=0
$$

where $\Delta \overline{u_{k}}$ is a properly defined stage-shifted time increment defined as $\Delta \overline{u_{k}}=\alpha_{k n} u^{n}+\sum_{j<k} \alpha_{k j} u_{j}$. For the two-stage RK scheme considered here we have

$$
\Delta \overline{u_{1}}=0 \text { and } \Delta \overline{u_{2}}=u_{1}-u^{n}
$$

Rearranging terms, and using the properties of the test function $w_{i}=\varphi_{i}+\gamma_{i}$ (cf. section $\S 1.2$, equations (28) and (29), one obtains

$$
\int_{\Omega} \varphi_{i}\left(\frac{\Delta u_{k}^{h}}{\Delta t}-\frac{\Delta{\overline{u_{k}}}^{h}}{\Delta t}\right)+\sum_{K \in \mathcal{D}_{i}} \sum_{j \in K} m_{i j}^{K}\left(\frac{\Delta \overline{u_{k}}}{\Delta t}\right)_{j}+\sum_{K \in \mathcal{D}_{i}} \beta_{i}^{K} \phi^{K(k)}=0
$$

having set

$$
\phi^{K(k)}=\int_{K} \nabla \cdot \boldsymbol{f}_{k}\left(u^{h}\right) d \boldsymbol{x}
$$


3. Mass lumping is applied to the Galerkin integrals in (56) (see e.g. 38 are references therein). Two possible choices are proposed in [10] and considered here. Either only the term containing the new values of the unknown is lumped, leading to the Selective Lumping (SL) formulation

$$
\left|S_{i}\right|\left(\frac{\Delta u_{k}}{\Delta t}\right)_{i}=-\sum_{K \in \mathcal{D}_{i}}\left(\Phi_{i}^{R K(k)}-\sum_{j \in K} m_{i j}^{G}\left(\frac{\Delta \overline{u_{k}}}{\Delta t}\right)_{j}\right)
$$

with $m_{i j}^{G}$ the Galerkin mass matrix, and with the short notation

$$
\Phi_{i}^{R K(k)}=\sum_{j \in K} m_{i j}^{K}\left(\frac{\Delta \overline{u_{k}}}{\Delta t}\right)_{j}+\beta_{i}^{K} \phi^{K(k)}
$$

A simpler update is obtained by lumping all the Galerkin integrals, which leads to the Global Lumping (GL) formulation

$$
\left|S_{i}\right|\left\{\left(\frac{\Delta u_{k}}{\Delta t}\right)_{i}-\left(\frac{\Delta \overline{u_{k}}}{\Delta t}\right)_{i}\right\}=-\sum_{K \in \mathcal{D}_{i}} \Phi_{i}^{R K(k)}
$$

The schemes obtained in this way are genuinely explicit, in the sense that no algebraic problem needs to be solved. For a more general derivation, and a truncation error analysis of the scheme obtained we refer to [10]. The specific form of the scheme is readily obtained by using the mass matrix and distribution coefficients presented in sections $\S 1.2$ and $\S 1.4$. For clarity, for each scheme, we will replace when necesary the superscript $R K(k)$ with $X X X(k)$ where $X X X$ is the shorthand notation for the scheme.

\section{Residual Distribution schemes for moving grids}

The objective of the following paragraph, and of the paper, is to recast the RK-RD schemes (57) and (58) in an ALE formalism. The starting point is the unstable Galerkin approximation of ALE equations presented in section $\S 1$. The discrete counterpart of the GCL naturally arise in the approximation. (but for a rigorous study about the implication of the GCL on the numerical scheme cf. [2, ?]): two different algorithms that likewise close the problem are presented. One is explained through the work of Dobes and Deconinck with a BDF2-RD scheme, the other consists basically in the approach due to Farhat for Explicit/Implicit Euler, Crank-Nicholson and Runge-Kutta time integrators. Then we move to stabilized Finite Elements and again we provide the extension of both the algorithms afromentioned. As a last step of the section the RK-RD approximation of the scalar ALE equations is presented.

\subsection{Galerkin Finite Element method}

We start with the approximation of $(20)$ both in time and space. The domain is initially approximated with an unstructured triangulation $\Omega_{h}^{X}$, then mapping 2 produce a time-continuous transformation of the grid $\Omega_{h}^{X} \rightarrow \Omega_{h}(t)$. The time discretization will make us evaluate the grid at instants $t^{n}$ generating a set of grid $\Omega_{h}\left(t^{n}\right)=\Omega_{h}^{n}$.

Moreover we ask our numerical method to satisfy a discrete version of the GCL condition (12), often referred to as Discrete Geometric Conservation Law (DGCL). Referring to the interpretation previously given, we are asking the method to preserve the state of uniform flow. We start with the simple Galerkin Finite Element space approximation which allows us a simple satisfaction of the GCL at a discrete level. 
We proceed in building the classical Galerkin method on the conservation law in ALE framework 20, with solution $u^{h}$, test function $\varphi_{i}$ and grid velocity $\boldsymbol{\sigma}^{h}=\sum_{j \in K} \varphi_{j} \boldsymbol{\sigma}_{j}$ belonging to the space of piecewise linear polynomials

$$
\int_{\Omega_{h}^{X}}\left(\left.\frac{\partial\left(J_{A} u^{h}\right)}{\partial t}\right|_{X}+J_{A} \nabla \cdot\left(\boldsymbol{f}\left(u^{h}\right)-\boldsymbol{\sigma}^{h} u^{h}\right)\right) \varphi_{i} d \boldsymbol{X}=0
$$

Since the the configuration $\Omega_{X}$ does not depend on time and assuming $\left.\frac{\partial \varphi_{i}}{\partial t}\right|_{X}=0$ we can take the time derivative outside the integral

$$
\left.\frac{\partial}{\partial t}\right|_{X} \int_{\Omega_{h}^{X}} \varphi_{i} J_{A} u^{h} d \boldsymbol{X}+\int_{\Omega_{X}} \varphi_{i} J_{A} \nabla \cdot\left(\boldsymbol{f}\left(u^{h}\right)-\boldsymbol{\sigma}^{h} u^{h}\right) d \boldsymbol{X}=0
$$

Passing to the current coordinates $\boldsymbol{x}$ we have the Galerkin approximation for 20

$$
\left.\frac{\partial}{\partial t}\right|_{X} \int_{\Omega_{h}(t)} \varphi_{i} u^{h} d \boldsymbol{x}+\int_{\Omega_{h}(t)} \varphi_{i} \nabla \cdot\left(\boldsymbol{f}\left(u^{h}\right)-\boldsymbol{\sigma}^{h} u^{h}\right) d \boldsymbol{x}=0
$$

If the flow is uniform we get the time continuous and space discrete approximation of 12 ,

$$
\begin{aligned}
\left.\frac{\partial}{\partial t}\right|_{X} \int_{\Omega_{h}(t)} \varphi_{i} d \boldsymbol{x} & =\int_{\Omega_{h}(t)} \varphi_{i} \nabla \cdot \boldsymbol{\sigma}^{h} d \boldsymbol{x} \\
\left.\sum_{K \in \mathcal{D}_{i}} \frac{1}{3} \frac{\partial K(t)}{\partial t}\right|_{X} & =\sum_{K \in \mathcal{D}_{i}} \frac{1}{3} \int_{K(t)} \nabla \cdot \boldsymbol{\sigma}^{h} d \boldsymbol{x}
\end{aligned}
$$

which reads as follows

$$
\left.\frac{\partial K(t)}{\partial t}\right|_{X}=\int_{K(t)} \nabla \cdot \boldsymbol{\sigma}^{h} d \boldsymbol{x}
$$

From w1 we clearly see that the satisfaction of the the GCL at a discrete level is related to the time scheme that one is using to integrate the conservation law. If one uses $\mathcal{B D} \mathcal{F} 3$ rather then $\mathcal{R} \mathcal{K} 2$ then, the different approximations of the left-hand side will lead to different ways of verifying exactly (61). We stress the fact that the DGCL is specific to the time scheme. If equation (61), approximated in time with the same scheme used to integrate the conservation law, is exactly satisfied, then the method is said to satisfy the Discrete Geometric Conservation Law.

\subsection{An example of a DGCL satisfying scheme}

A very useful time integrator is $\mathcal{B D} \mathcal{F} 2$ which provide second order accuracy in time. We explain how to satisfy the DGCL for $\mathcal{B D} \mathcal{F} 2$ following a method proposed by Dobes in a $\mathcal{R} \mathcal{D}$ framework 9 . The weak form is obtained starting from a sligthly different form of 20 obtained by splitting the ALE flux term and using the fact that $\boldsymbol{a}=\boldsymbol{a}(u)$

$$
\left.\frac{\partial\left(J_{A} u\right)}{\partial t}\right|_{X}+J_{A}(\boldsymbol{a}-\boldsymbol{\sigma}) \nabla \cdot u-J_{A} u \nabla \cdot \boldsymbol{\sigma}=0
$$

Since we want to satisfy the GCL condition we substitute 12 into the above equation

$$
\left.\frac{\partial}{\partial t}\right|_{X} \int_{\Omega_{X}} J_{A} \varphi_{i} u_{h} d \boldsymbol{X}+\int_{\Omega_{X}} J_{A} \varphi_{i}\left(\boldsymbol{a}\left(u^{h}\right)-\boldsymbol{\sigma}^{h}\right) \cdot \nabla u^{h} d \boldsymbol{X}-\left.\frac{\partial J_{A}}{\partial t}\right|_{X} \int_{\Omega_{X}} \varphi_{i} u_{h} d \boldsymbol{X}=0
$$


The last term is usually reffered to as Geometric Source Term. After some calculation the algorithm is rewritten

$$
\left.\sum_{K \in \mathcal{D}_{i}} \sum_{j \in K} \hat{m}_{i j}^{G} \frac{\partial\left(|K| u_{j}\right)}{\partial t}\right|_{X}+\int_{\Omega_{h}(t)} \varphi_{i}\left(\boldsymbol{a}\left(u^{h}\right)-\boldsymbol{\sigma}^{h}\right) \cdot \nabla u^{h} d \boldsymbol{x}-\left.\sum_{K \in \mathcal{D}_{i}} \frac{\partial|K|}{\partial t}\right|_{X} \sum_{j \in K} \hat{m}_{i j}^{G} u_{j}=0
$$

where $\hat{m}_{i j}^{G}=\frac{\delta_{i j}}{4}+\frac{1}{4}$ is the Galerkin mass matrix. As we can see, satisfying the GCL is completely different from satisfing the DGCL for which we have to discretize both the derivatives with the same time discrete operator. Proceeding in this fashion we are sure to balance, element by element, the volume variation in the time step with the integral of the grid velocity flux along the boundaries of the element 61.

This approach has a nice recasting into a $\mathcal{R D}$ framework. In fact the second term in $(63)$ is already in a quasi-linear form, so it can be written in a $\mathcal{R} \mathcal{D}$ form through a conservative linearization. Since the grid velocity is approximated with $\mathbb{P}^{1}$ interpolation the correct conservative linearization of the ALE part is immediate

$$
\int_{\Omega(t)} \varphi_{i} \boldsymbol{\sigma}^{h} \cdot \nabla u^{h} d \boldsymbol{x}=\sum_{j \in K} \frac{\boldsymbol{\sigma}_{j}}{3} \nabla u^{h}|K|=\overline{\boldsymbol{\sigma}} \nabla u^{h}|K|
$$

The upwind parameter with the ALE correction naturally becomes

$$
k_{i}=\frac{1}{2}(\overline{\boldsymbol{a}}-\overline{\boldsymbol{\sigma}}) \cdot \boldsymbol{n}_{i}
$$

If $\alpha^{n+1}, \alpha^{n}, \alpha^{n-1}$ are the coefficients of $\mathcal{B D} \mathcal{F} 2$, the Galerkin $\mathcal{R D}$ scheme then reads

$$
\begin{gathered}
\sum_{K \in \mathcal{D}_{i}} \sum_{j \in K} \hat{m}_{i j}^{G} \frac{\alpha^{n+1}\left|K^{n+1}\right| u_{j}^{n+1}+\alpha^{n}\left|K^{n}\right| u_{j}^{n}+\alpha^{n-1}\left|K^{n-1}\right| u_{j}^{n-1}}{\Delta t}+\sum_{K \in \mathcal{D}_{i}} \beta_{i}^{G} \sum_{j \in K} k_{j}^{n+1} u_{j}^{n+1}+ \\
-\sum_{K \in \mathcal{D}_{i}} \frac{\alpha^{n+1}\left|K^{n+1}\right|+\alpha^{n}\left|K^{n}\right|+\alpha^{n-1}\left|K^{n-1}\right|}{\Delta t} \sum_{j \in K} \hat{m}_{i j}^{G} u_{j}=0
\end{gathered}
$$

where $\beta^{G}=\frac{1}{3}$ is the distribution coefficient for the Galerkin method. Relation 65 satisfies the DGCL by construction. This is supposed to be just an example since Galerkin method for hyperbolic problems is unstable.

\subsection{An approach "à la Farhat"}

Keeping in mind that our objective is a method verifying the time discrete counterpart of (61), in this paragraph we proceed in a different way, according to what suggested by Farhat in [39]. The main idea is that many of the most used time discretizations satisfy naturally the DGCL condition by the choice of a proper grid velocity and of a proper quadrature rule for the integrals.

First, we present some useful results that will be use everywhere hereinafter. Integrating (61) in the timeslab $\left[t^{n}, t^{n+1}\right]$ provides

$$
|K|^{n+1}-|K|^{n}=\int_{t^{n}}^{t^{n+1}} \int_{\partial K(t)} \nabla \cdot \boldsymbol{\sigma}^{h} d \boldsymbol{x} d t
$$


We have already observed that great care has to be put, when building the numerical method, in order to satisfy exactly the above equation. This can be done with simple geometry and algebra. Since the triangle area can be computed as $|K|=\frac{1}{2} \sum_{j \in K} \boldsymbol{x}_{j} \cdot \boldsymbol{k}_{j}$ with

$$
\begin{aligned}
|K|^{n+1}-|K|^{n} & =\frac{1}{2} \sum_{j \in K}\left[\left(\boldsymbol{x}_{j} \cdot \boldsymbol{k}_{j}\right)^{n+1}-\left(\boldsymbol{x}_{j} \cdot \boldsymbol{k}_{j}\right)^{n}\right] \\
& =\frac{1}{2} \sum_{j \in K}\left[\boldsymbol{x}_{j}^{n+1 / 2} \cdot\left(\boldsymbol{k}_{j}^{n+1}-\boldsymbol{k}_{j}^{n}\right)+\boldsymbol{k}_{j}^{n+1 / 2} \cdot\left(\boldsymbol{x}_{j}^{n+1}-\boldsymbol{x}_{j}^{n}\right)\right] \\
& =\sum_{j \in K} \boldsymbol{k}_{j}^{n+1 / 2} \cdot\left(\boldsymbol{x}_{j}^{n+1}-\boldsymbol{x}_{j}^{n}\right)
\end{aligned}
$$

If we set the grid velocity

$$
\boldsymbol{\sigma}_{j}^{*}=\frac{\boldsymbol{x}_{j}^{n+1}-\boldsymbol{x}_{j}^{n}}{\Delta t}
$$

We can recast (67) in the following form

$$
|K|^{n+1}-|K|^{n}=\Delta t \int_{K^{n+1 / 2}} \nabla \cdot \boldsymbol{\sigma}_{h}^{*} d \boldsymbol{x}
$$

We have proved that, in order to satisfy (66), a natural choice for the grid velocity is 68 while the configuration on which we perform integrations should be the midpoint one between $t^{n}$ and $t^{n+1}$.

We found the result of 39 for which it is crucial to establish in 66 where the time integral must be computed and the same question arise for the grid velocity. Since the left-end side is always computed exactly, an appropriate scheme for evaluating exactly $\int_{t^{n}}^{t^{n+1}} \int_{\Omega_{h}} \nabla \cdot \boldsymbol{\sigma}_{h} d \boldsymbol{x} d t$ is presented

$$
\int_{t^{n}}^{t^{n+1}} \int_{K} \nabla \cdot \boldsymbol{\sigma}_{h} d \boldsymbol{x} d t=\Delta t \int_{K^{n+\frac{1}{2}}} \nabla \cdot \boldsymbol{\sigma}_{h}^{*} d \boldsymbol{x}
$$

This result is very useful once we have discretized in time 60.

\subsubsection{Explicit Euler}

Discretizing in time 60 with an explicit Euler tme discretisation, we have

$$
\frac{\Delta}{\Delta t} \int_{\Omega_{h}(t)} \varphi_{i} u_{h} d \boldsymbol{x}+\int_{\Omega_{h}(t)} \varphi_{i} \nabla \cdot\left(\boldsymbol{f}\left(u_{h}^{n}\right)-\boldsymbol{\sigma}^{h} u_{h}^{n}\right) d \boldsymbol{x}=0
$$

We have still to face the problem of satisfying the DGCL, both $\boldsymbol{\sigma}$ and $\Omega_{h}(t)$ are undefined infact. Imposing a uniform flow, one sees that the satisfaction of the DGCL conditon passes through 69 . Setting $\boldsymbol{\sigma}_{j}=\boldsymbol{\sigma}_{j}^{*}$ and $\Omega_{h}(t)=\Omega_{h}^{n+1}$ we close the problem

$$
\int_{\Omega_{h}^{n+1}} \varphi_{i} u_{h}^{n+1} d \boldsymbol{x}-\int_{\Omega_{h}^{n}} \varphi_{i} u_{h}^{n} d \boldsymbol{x}+\Delta t \int_{\Omega_{h}^{n+1 / 2}} \varphi_{i} \nabla \cdot\left(\boldsymbol{f}\left(u_{h}^{n}\right)-\boldsymbol{\sigma}_{h}^{*} u_{h}^{n}\right) d \boldsymbol{x}=0
$$




\subsubsection{Implicit Euler}

We proceed as before, setting $\boldsymbol{\sigma}_{j}=\boldsymbol{\sigma}_{j}^{*}$ and $\Omega_{h}(t)=\Omega_{h}^{n+1}$ and we found that the DGCL condition is again satisfied.

$$
\int_{\Omega_{h}^{n+1}} \varphi_{i} u_{h}^{n+1} d \boldsymbol{x}-\int_{\Omega_{h}^{n}} \varphi_{i} u_{h}^{n} d \boldsymbol{x}+\Delta t \int_{\Omega_{h}^{n+1}} \varphi_{i} \nabla \cdot\left(\boldsymbol{f}\left(u_{h}^{n+1}\right)-\boldsymbol{\sigma}_{h}^{*} u_{h}^{n+1}\right) d \boldsymbol{x}=0
$$

In fact imposing a uniform flow, one finds 69 .

\subsubsection{Crank-Nicholson}

Also in this case, the imposition of a uniform flow leads to equation 69 which is exactly satisfied for $\boldsymbol{\sigma}_{j}=\boldsymbol{\sigma}_{j}^{*}$ and $\Omega_{h}(t)=\Omega_{h}^{n+1}$. Hence we have

$$
\begin{array}{r}
\int_{\Omega_{h}^{n+1}} \varphi_{i} u_{h}^{n+1}-\int_{\Omega_{h}^{n}} \varphi_{i} u_{h}^{n}+\frac{\Delta t}{2}\left(\int_{\Omega_{h}^{n+1}} \varphi_{i} \nabla \cdot\left(\boldsymbol{f}\left(u_{h}^{n+1}\right)-\boldsymbol{\sigma}_{h}^{*} u_{h}^{n+1}\right)\right. \\
\left.+\int_{\Omega_{h}^{n+1}} \varphi_{i} \nabla \cdot\left(\boldsymbol{f}\left(u_{h}^{n}\right)-\boldsymbol{\sigma}_{h}^{*} u_{h}^{n}\right)\right)=0 .
\end{array}
$$

\subsection{Stabilized Finite Elements and Residual Distribution}

The Galerkin method provide a centered approximation of the advective part leading to an unstable numerical solution. To cure this problem the stabilized Finite Element method is invoked in this paragraph. We consider now the addition to the Galerkin scheme of a proper stabilization operator, which we shall denote by $\mathcal{L}_{h}$, depending on some bubble function $\gamma_{i}$ (cf. $\$ 1.2$ equation (27) and sub-sequent text). Several choices are possible

1. Using the conservation law in conservative form 20 one gets

$$
\mathcal{L}_{h}=\sum_{K} \int_{K_{X}} \gamma_{i}\left(\left.\frac{\partial\left(J_{A} u\right)}{\partial t}\right|_{X}+J_{A} \nabla \cdot(\boldsymbol{f}-u \boldsymbol{\sigma})\right) d \boldsymbol{X}
$$

2. Using the mixed formulation 21

$$
\mathcal{L}_{h}=\sum_{K} \int_{K} \gamma_{i}\left(\left.\frac{\partial u}{\partial t}\right|_{X}+\nabla \cdot \boldsymbol{f}-\boldsymbol{\sigma} \cdot \nabla u\right) d \boldsymbol{x}
$$

3. 39] within a Finite Element method in [39] uses instead the Eulerian formulation

$$
\mathcal{L}_{h}=\sum_{K} \int_{K} \gamma_{i}\left(\frac{\partial u}{\partial t}+\nabla \cdot \boldsymbol{f}\right) d \boldsymbol{x}
$$

In the next section we show two different ways of formulating a GCL-satisfying stabilized FE- $\mathcal{R D}$ using 73. as stabilization term. 


\subsubsection{Dobes Closure for explicit Euler time stepping}

We can rearrange the weak form as

$$
\int_{\Omega_{h}^{X}}\left(\left.\frac{\partial\left(J_{A} u^{h}\right)}{\partial t}\right|_{X}+J_{A} \nabla \cdot\left(\boldsymbol{f}\left(u^{h}\right)-\boldsymbol{\sigma}^{h} u^{h}\right)\right) w_{i} d \boldsymbol{X}=0 \quad \forall i \in \mathcal{T}_{h}
$$

where the test function is $w_{i}=\varphi_{i}+\gamma_{i}$.

We get

$$
\left.\sum_{K \in \mathcal{D}_{i}} \sum_{j \in K} \hat{m}_{i j}^{K} \frac{\partial\left(|K| u_{j}\right)}{\partial t}\right|_{X}+\int_{\Omega(t)} w_{i}\left(\boldsymbol{a}\left(u^{h}\right)-\boldsymbol{\sigma}^{h}\right) \cdot \nabla u^{h} d \boldsymbol{x}-\left.\sum_{K \in \mathcal{D}_{i}} \frac{\partial|K|}{\partial t}\right|_{X} \sum_{j \in K} \hat{m}_{i j}^{K} u_{j}=0
$$

where $\hat{m}_{i j}^{K}$ is the general mass matrix that depends on the test function, introduced in section 3.2 . Discretizing the time derivative with Explict Euler, lumping the mass-matrix, and using the FEM-RD analogy

$$
\frac{\left|S_{i}^{n+1}\right| u_{i}^{n+1}-\left|S_{i}^{n}\right| u_{i}^{n}}{\Delta t}+\sum_{K \in \mathcal{D}_{i}} \beta_{i} \sum_{j \in K} k_{j}^{n} u_{j}^{n}-\frac{\left|S_{i}^{n+1}\right|-\left|S_{i}^{n}\right|}{\Delta t} u_{i}^{n}=0
$$

For the presence of the Geometric Source Term, the above scheme is not written in the compact prototype form but one can prove that a sub-element positivity property still holds. The scheme for a single element is written

$$
\begin{gathered}
\frac{\left|K^{n+1}\right| u_{i}^{n+1}-\left|K^{n}\right| u_{i}^{n}}{3 \Delta t}+\sum_{j \in K} c_{i j}^{K} u_{j}^{n}-\frac{\left|K^{n+1}\right|-\left|K^{n}\right|}{3 \Delta t} u_{i}^{n}=0 \\
u_{i}^{n+1}=\left(\frac{\left|K^{n}\right|+\left|K^{n+1}\right|}{\left|K^{n+1}\right|}-\frac{3 \Delta t}{\left|K^{n+1}\right|} c_{i i}^{K}\right) u_{i}^{n}-\frac{3 \Delta t}{\left|K^{n+1}\right|} \sum_{j \in K, j \neq i} c_{i j}^{K} u_{j}^{n}
\end{gathered}
$$

Positivity is ensured with the following CFL-like condition

$$
\Delta t \leq \frac{\left|K^{n}\right|+\left|K^{n+1}\right|}{3 c_{i i}^{K}} \quad \forall i, K \in \mathcal{T}_{h}
$$

Dobes used this approach together with second order implicit time schemes, in particular BDF2 with consistent mass-matrix - getting the stabilized version of the algorithm of paragraph (2.2.2) - and CrankNicholson with lumped mass matrix, obtaining very good results. We have to mention only that, if a consistent mass-matrix formulation is used, then positivity is spoiled.

\subsubsection{Another closure for explicit Euler time stepping}

We suggest another closure to the problem which is somewhat simpler. We start again from equation (76) discretized in time with Euler-Explicit time stepping, we split again the ALE flux term, finally we use grid velocity 68 and midpoint configuration.

$$
\frac{\Delta}{\Delta t} \int_{\Omega(t)} w_{i} u^{h} d \boldsymbol{x}+\int_{\Omega^{n+1 / 2}} w_{i}\left(\nabla \cdot \boldsymbol{f}\left(u_{h}^{n}\right)-\boldsymbol{\sigma}_{h}^{*} \cdot \nabla u_{h}^{n}\right) d \mathbf{x}-\int_{\Omega^{n+1 / 2}} w_{i} u_{h}^{n} \nabla \cdot \boldsymbol{\sigma}_{h}^{*} d \boldsymbol{x}=0
$$

If a uniform flow is imposed one gets

$$
\int_{\Omega_{h}^{n+1}} w_{i} d \boldsymbol{x}-\int_{\Omega_{h}^{n}} w_{i} d \boldsymbol{x}=\Delta t \int_{\Omega_{h}^{n+1 / 2}} w_{i} \nabla \cdot \boldsymbol{\sigma}_{h}^{*} d \boldsymbol{x}
$$


It is easy to check that the above equation is verified exactely. In fact for property (28) we have

$$
\sum_{K \in \mathcal{D}_{i}} \beta_{i}^{K}\left(|K|^{n+1}-|K|^{n}\right)=\Delta t \sum_{K \in \mathcal{D}_{i}} \beta_{i}^{K} \int_{\Omega_{h}^{n+1 / 2}} \nabla \cdot \boldsymbol{\sigma}_{h}^{*} d \boldsymbol{x}
$$

Thus, we get again 69 which is an identity.

$$
\begin{aligned}
\left(\int_{\Omega(t)} w_{i} u^{h} d \boldsymbol{x}\right)^{n+1}- & \left(\int_{\Omega(t)} w_{i} u^{h} d \boldsymbol{x}\right)^{n}= \\
& \int_{\Omega^{n+1 / 2}} w_{i}\left(u_{h}^{n+1}-u_{h}^{n}\right) d \boldsymbol{x}+\Delta t \int_{\Omega^{n+1 / 2}} w_{i} \frac{\left(u_{h}^{n+1}+u_{h}^{n}\right)}{2} \nabla \cdot \boldsymbol{\sigma}_{h}^{*} d \boldsymbol{x}
\end{aligned}
$$

Substituting $(79)$ in $(78)$ and we sum the last term of the above equation with the last one in 78

$$
\begin{aligned}
\left(1+\frac{\Delta t}{2} \nabla \cdot \boldsymbol{\sigma}_{h}^{*}\right) \int_{\Omega^{n+1 / 2}} w_{i} & \left(u_{h}^{n+1}-u_{h}^{n}\right) d \boldsymbol{x} \\
& +\Delta t \int_{\Omega^{n+1 / 2}} w_{i}\left(\nabla \cdot \boldsymbol{f}\left(u_{h}^{n}\right)-\boldsymbol{\sigma}_{h}^{*} \cdot \nabla u_{h}^{n}\right) d \boldsymbol{x}=0
\end{aligned}
$$

Finally, using the analogy with Residual Distribution method, and lumping the Galerkin mass matrix :

$$
\sum_{K \in \mathcal{D}_{i}}\left(1+\frac{\Delta t}{2} \nabla \cdot \boldsymbol{\sigma}_{h}^{*}\right) \frac{\left|K^{n+1 / 2}\right|}{3}\left(u_{i}^{n+1}-u_{i}^{n}\right)=-\Delta t \sum_{K \in \mathcal{D}_{i}} \beta_{i} \sum_{j} k_{j}^{n} u_{j}^{n}
$$

And the final algorithm reads

$$
\left|\tilde{S}_{i}^{n+1 / 2}\right|\left(u_{i}^{n+1}-u_{i}^{n}\right)=-\Delta t \sum_{K \in \mathcal{D}_{i}} \beta_{i} \sum_{j} k_{j}^{n} u_{j}^{n}
$$

where the median dual cell area of 39 , here evaluated at midpoint configuration, has to be modified to take into account the grid distortion

$$
\left|\tilde{S}_{i}^{n+1 / 2}\right|=\sum_{K \in \mathcal{D}_{i}}\left(1+\frac{\Delta t}{2} \nabla \cdot \boldsymbol{\sigma}_{h}^{*}\right) \frac{\left|K^{n+1 / 2}\right|}{3}
$$

The method satisfies the DGCL by construction and it is extremely easy to prove this again, by simply assuming a uniform state in the method presented so far.

Apart from the ALE flux part in the upwind parameter $k_{j}$, the formulation follows very closely the prototype scheme in Eulerian formulation, an extension of the results regarding positivity should be straightforward. A modified median dual cell area appear to take into account mesh distortion $\left(1+\frac{\Delta t}{2} \nabla \cdot \boldsymbol{\sigma}_{h}^{*}\right)$. Strictly speaking this scalar quantity can be also negative (in a critical situation of very fast compression for the mesh) spoiling every positive coefficient analysis. In practice, if the grid displacements within the time step are of order $h$ then this term is of order $\mathcal{O}\left(h^{2}\right)$ and does not affect the positivity properties of the scheme.. In all the computations performed and reported in the following sections, even the ones involving large mesh distortion, the positivity of $\left|\tilde{S}_{i}^{n+1 / 2}\right|$ was always maintained. 


\subsection{Two-stage RK- $\mathcal{R} \mathcal{D}$ time stepping}

A direct extension of the method described to the RK- $\mathcal{R} \mathcal{D}$ time marching of section $\S 2$ cannot be performed. The problem that we have to face is related to the definitions of the stage-shifted time increment (55) which, in the first step, is zero. This breaks the terms balance leading to the breaking of the DGCL. A simple way to fix this inconvenient is to carefully modify the stabilization term equation (74). In particular, the Galerkin part discretized with a $\mathcal{R} \mathcal{K} 2$, with $\boldsymbol{\sigma}^{h}=\boldsymbol{\sigma}_{h}^{*}$ and midpoint configuration, writes

$$
\begin{aligned}
\frac{\Delta}{\Delta t} \int_{\Omega(t)} \varphi_{i} u_{k}^{h} d \boldsymbol{x} & +\int_{\Omega^{n+1 / 2}} \varphi_{i}\left(\nabla \cdot \boldsymbol{f}\left(u^{h}\right)-\boldsymbol{\sigma}_{h}^{*} \cdot \nabla u^{h}\right)_{k} d \boldsymbol{x} \\
& -\int_{\Omega^{n+1 / 2}} \varphi_{i}\left(u^{h} \nabla \cdot \boldsymbol{\sigma}_{h}^{*}\right)_{k} d \boldsymbol{x}=0
\end{aligned}
$$

For the stabilization one, also computed at midpoint configuration, we have

$$
\sum_{K \in \mathcal{D}_{i}} \int_{K^{n+1 / 2}} \gamma_{i} \frac{\Delta{\overline{u_{k}}}^{h}}{\Delta t} d \boldsymbol{x}+\sum_{K \in \mathcal{D}_{i}} \int_{K^{n+1 / 2}} \gamma_{i}\left(\nabla \cdot \boldsymbol{f}\left(u^{h}\right)^{n}-\boldsymbol{\sigma}_{h}^{*} \cdot \nabla u^{h}\right)_{k} d \boldsymbol{x}=0
$$

Now, in analogy with the notation introduced in section $\S 2$, we set

$$
\Phi_{i}^{R K(k)}=\int_{K^{n+1 / 2}} w_{i}\left(\frac{\Delta{\overline{u_{k}}}^{h}}{\Delta t}+\left(\nabla \cdot \boldsymbol{f}\left(u^{h}\right)-\boldsymbol{\sigma}_{h}^{*} \nabla u^{h}\right)_{k}\right) d \boldsymbol{x}
$$

or equivalently

$$
\Phi_{i}^{R K(k)}=\sum_{j \in K} m_{i j}^{K}\left(\frac{\Delta \overline{u_{k}}}{\Delta t}\right)_{j}+\beta_{i}^{K} \phi^{K(k)}
$$

In particular exploiting the two RK steps read

$$
\begin{cases}\Phi_{i}^{R K(1)}= & \phi_{i}\left(u^{h}\right)^{n} \\ \Phi_{i}^{R K(2)}=\sum_{j \in K} m_{i j}^{K} \frac{u_{j}^{1}-u_{j}^{n}}{\Delta t} & +\frac{1}{2}\left(\phi_{i}\left(u^{h}\right)_{1}+\phi_{i}\left(u^{h}\right)^{n}\right)\end{cases}
$$

Finally we can give the following result of which we report the proof in appendix $\mathrm{A}$.

Proposition 4.1 (Second order two stage ALE RK-RD schemes) The DGCL satisfying ALE formulation of schemes (57) and (58) is defined by

$$
\left|\tilde{S}_{i}^{n+1 / 2}\right| \frac{\Delta u_{i}^{k}}{\Delta t}=-\sum_{K}\left(\Phi_{i}^{R K(k)}-\sum_{j} \tilde{m}_{i j}^{G} \frac{\Delta \overline{u_{j}^{k}}}{\Delta t}\right)
$$

in the Selective Lumping case, with

$$
\tilde{m}_{i j}^{G}=\left(1+\frac{\Delta t}{2} \nabla \cdot \boldsymbol{\sigma}_{h}^{*}\right) m_{i j}^{G}
$$

and

$$
\left\{\begin{array}{l}
\left|\tilde{S}_{i}^{n+1 / 2}\right| \frac{u_{i}^{1}-u_{i}^{n}}{\Delta t}=-\sum_{K} \Phi_{i}^{R K(1)} \\
\left|\tilde{S}_{i}{ }^{n+1 / 2}\right| \frac{u_{i}^{n+1}-u_{i}^{1}}{\Delta t}=-\sum_{K} \Phi_{i}^{R K(2)}
\end{array}\right.
$$

in the Global Lumping case. 
Apart from the ALE part in the definition of the upwind parameter, the only differences respect to Eulerian version (57) is the presence of the modified median dual cell area (82) and of a modified Galerkin mass matrix, while for Global Lumping we obtain a very similar expression to (58), with the exception of the modification of the median dual cell area.

The following sections are devoted to the numerical assessment of the accuracy and robustness of the schemes obtained for both scalar problems and for the perfect gas Euler equations.

\section{Numerical results for scalar problems}

The scalar experiments we show here are used to test the formulation presented so far. The test cases are exactely the ones performed by [10] with an explicit RK2 scheme: we expect to recover the same results, in terms of accuracy and non-oscillatory behaviour, when the grid is moving with an arbitrary motion and the RK2-ALE scheme presented in the previous section is used.

All the schemes, modified in the proper way for RK2 time integrator of section $\S 2$ are used here. We have just to remember that, for ALE computations, the upwind parameter takes into account the grid movement and follows the definition 64 which is recalled below

$$
k_{i}=\frac{1}{2}(\overline{\boldsymbol{a}}-\overline{\boldsymbol{\sigma}}) \cdot \boldsymbol{n}_{i}
$$

All the definition which involves $k_{i}$ has been revisted.

For all the experiments the time step is computed in order to verify the CFL condition

$$
\Delta t=\mathrm{CFL} \min _{i \in \mathcal{T}_{h}} \frac{\left|S_{i}\right|}{\sum_{K \in \mathcal{D}_{i}} \alpha^{K}}
$$

where $\mathrm{CFL}=0.8$ has been adopted.

\subsection{Convergence properties}

To test the accuracy of the method we use the simple case of linear advection of a smooth sinusoidal hill

$$
\begin{cases}\frac{\partial u}{\partial t}+\boldsymbol{a} \cdot \nabla u=0, & \boldsymbol{a}=[0,1], \boldsymbol{x} \in[0,1] \times[0,2], t \in[0,1] \\ u_{0}=\cos (2 \pi r) & \text { if } r \leq 0.25, r=\sqrt{(x-0.5)^{2}+(y-0.5)^{2}} \\ u_{0}=0 & \text { otherwise }\end{cases}
$$

We choose four unstructured grid with characteristic lenghts $h \in\{1 / 30,1 / 50,1 / 80,1 / 160\}$. The reference domain $\boldsymbol{X}=(X, Y)$ is mapped according to

$$
\boldsymbol{x}=\boldsymbol{X}+\sin (2 \pi X) \sin (\pi Y)(0.1 \sin (2 \pi t), 0.2 \sin (4 \pi t))
$$

At $t=1$, the mapping is the identity $\boldsymbol{x}=\boldsymbol{X}$, so we can compare the ALE solution with the Eulerian one easily. In figure 2 , the third grid $(h=1 / 80)$ is shown with the correspondent mapping.

All the results collected in figure 3 shows that second order of accuracy, when expected, is achieved. The ALE convergence curve almost collapse on the classical one. The Blended LDA-N on smooth solution should collapse to the LDA scheme but it converges more slowly, only with order 1.5 instead. This is due to the fact that the advecting hill is very narrow and the presence of strong gradients cause the switch to a first order $\mathrm{N}$ scheme. 

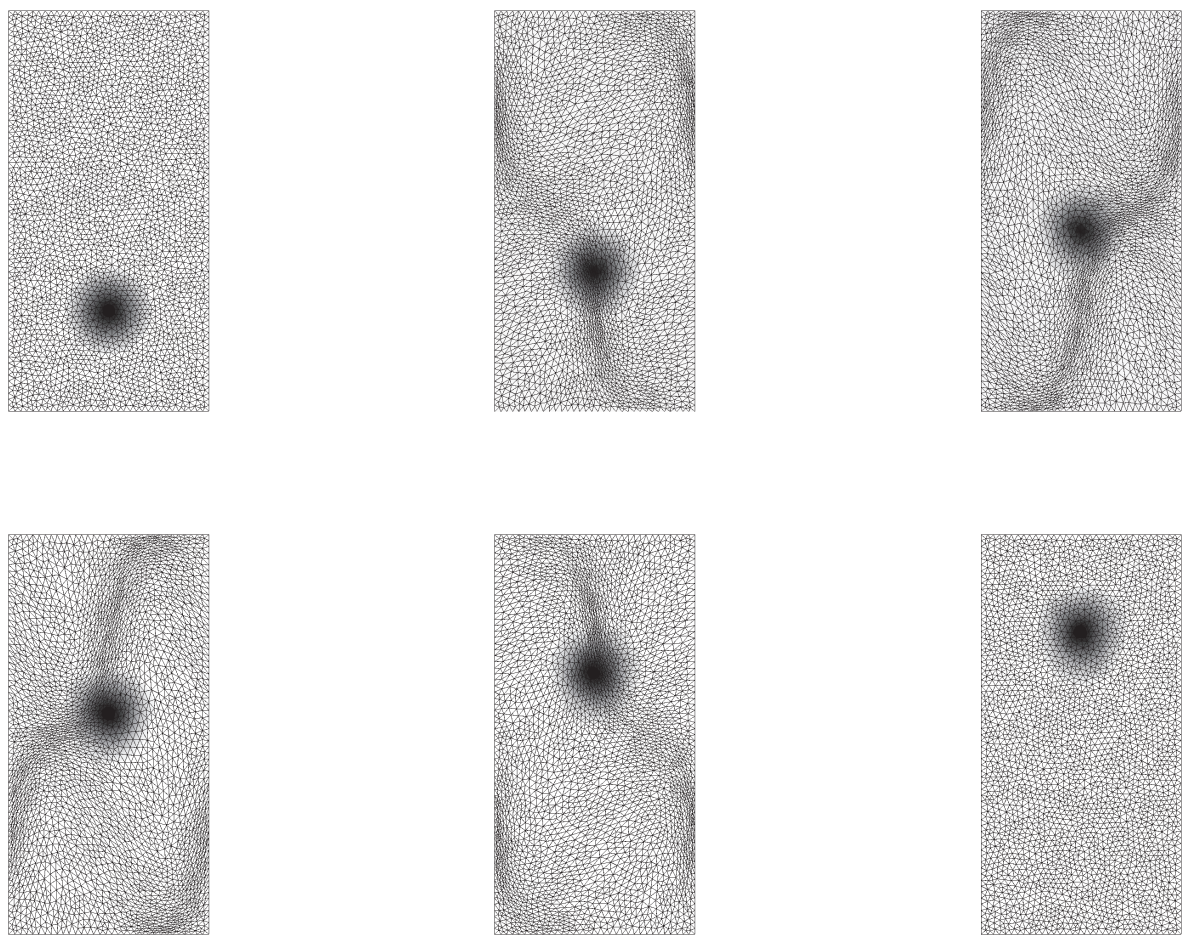

Figure 2: Linear Advection. Mapping for the grid and example of the numerical solution

\subsection{D Burger equation}

The non-linear Burger equation is a good test to see how the schemes behaves near discontinuities

$$
\begin{cases}\frac{\partial u}{\partial t}+\boldsymbol{a} \cdot \nabla u=0, & \boldsymbol{a}=[u, u], x \in[-1,1] \times[-1,1], t \in[0,1] \\ u_{0}=1 & \text { if } x \in[-0.6,-0.1] \times[-0.35,0.15] \\ u_{0}=0 & \text { otherwise }\end{cases}
$$

The reference grid size is $h=1 / 80$. The domain is mapped in a similar way as (88), according to

$$
\boldsymbol{x}=\boldsymbol{X}+0.2 \sin (\pi X) \sin (\pi Y)(\sin (2 \pi t), \sin (4 \pi t))
$$

Only results with formulation F1 are shown but the use of formulation F2 leads to very similar results.

First we consider the linearity preserving LDA and SUPG scheme in figures 4,5 , 6 and 7 . As expected, these two schemes gives very good results when computing smooth solutions, and fails when computing discontinuities. Oscillations appears on the shock and at the tail of the rarefaction wave. The important observation is that the ALE results, far from the discontinuity, are very close to the Eulerian ones, on the tail of the rarefaction even better.

The non-linear schemes LLxFs and LDA-N are designed to capture discontinuities very well. This is shown in figures $8,9,10$ and 11. We have seen that SL formulation, unfortunately, does not allow us 

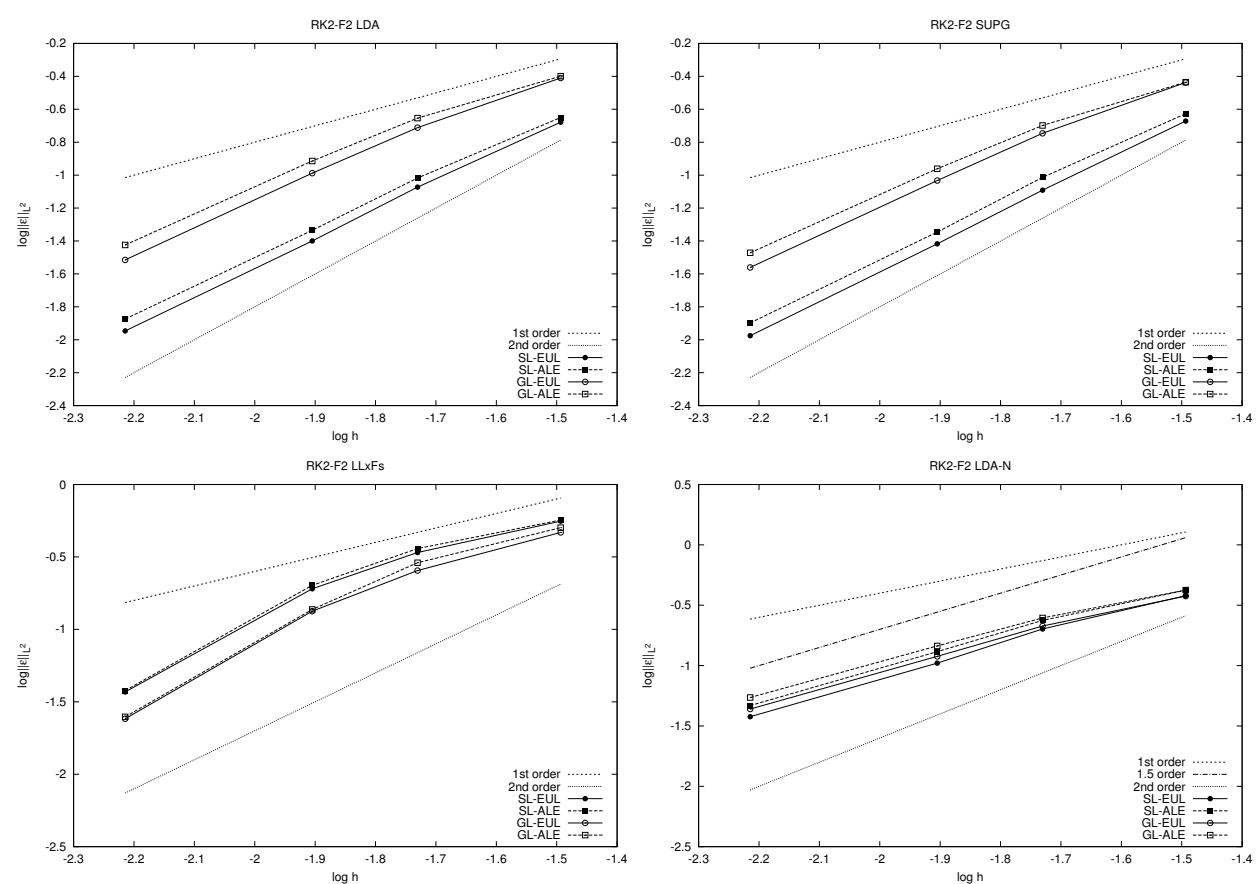

Figure 3: Linear Advection. Order of Convergence

to maintain positivity hence oscillation still appear but are less pronounced compared to linear schemes. With GL formulation this problem is cured provided that some dissipation is introduced due to masslumping [10. The ALE results reproduce very well the Eulerian ones and they are even better for the tail of the rarefaction wave.
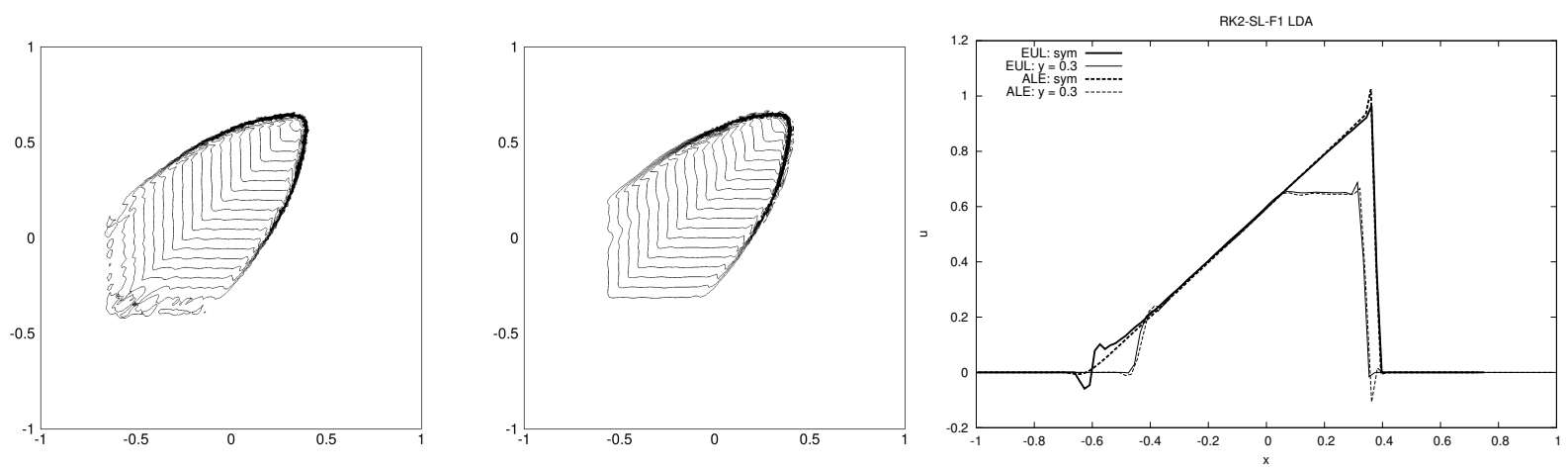

Figure 4: 2D Burger Equation, F1-SL LDA scheme. Left and middle: 20 equispaced isolines between 0 and 1 at time $t=1$. left, Eulerian. right ALE. Right: comparison of the solution along the symmetry line and along the line $y=0.3$ 

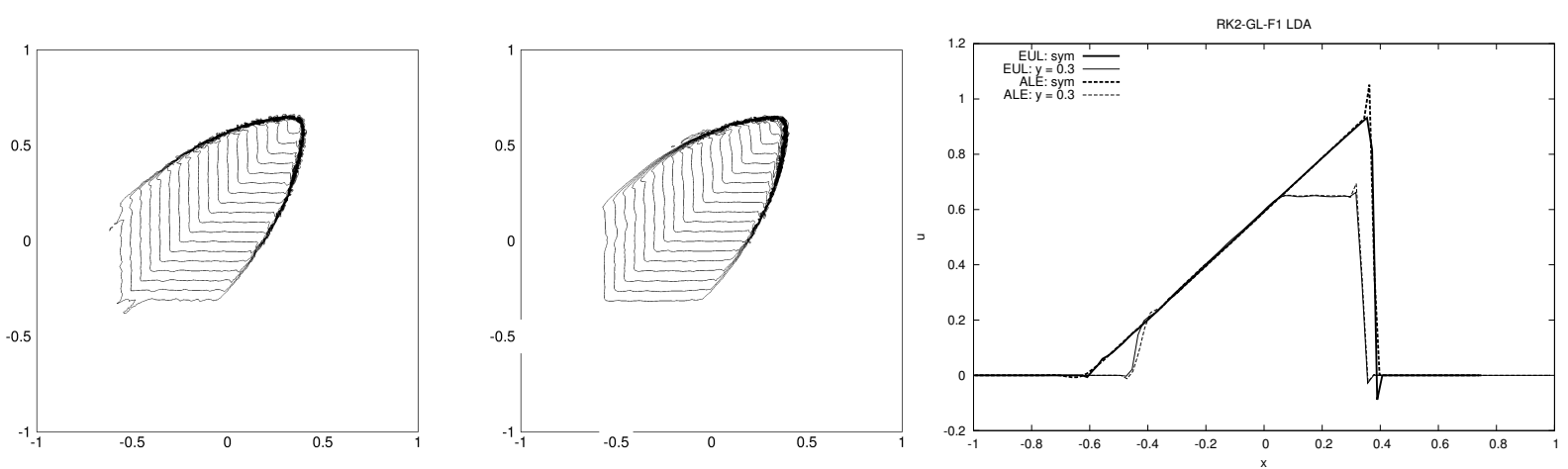

Figure 5: 2D Burger Equation, F1-GL LDA scheme. Left and middle: 20 equispaced isolines between 0 and 1 at time $t=1$. left, Eulerian. right ALE. Right: comparison of the solution along the symmetry line and along the line $y=0.3$
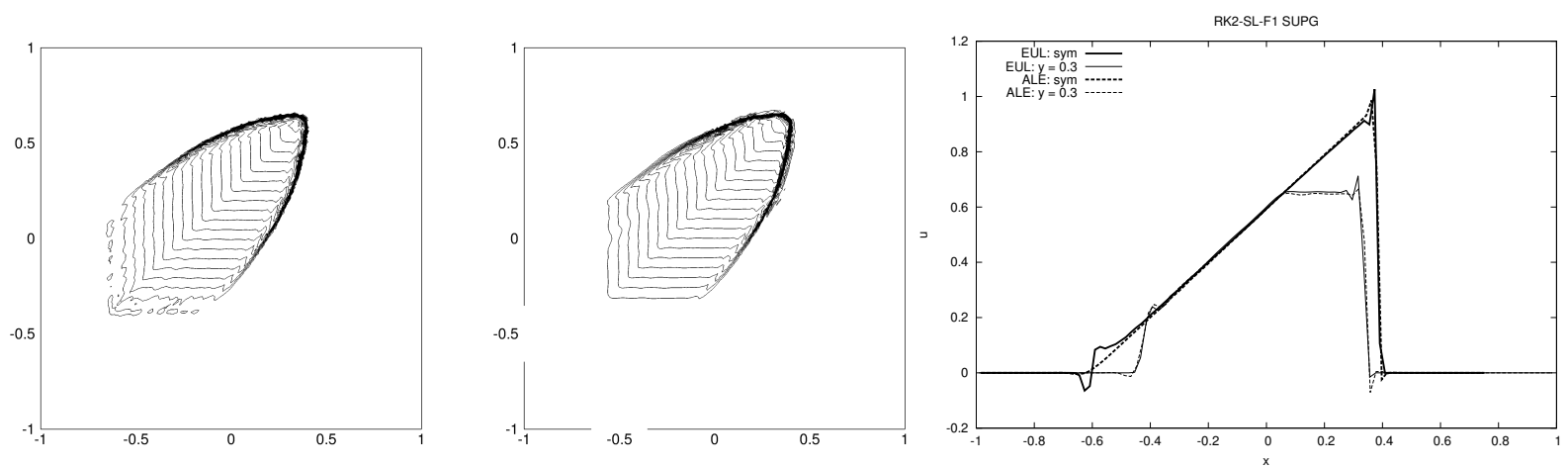

Figure 6: 2D Burger Equation, F1-SL SUPG scheme. Left and middle: 20 equispaced isolines between 0 and 1 at time $t=1$. left, Eulerian. right ALE. Right: comparison of the solution along the symmetry line and along the line $y=0.3$
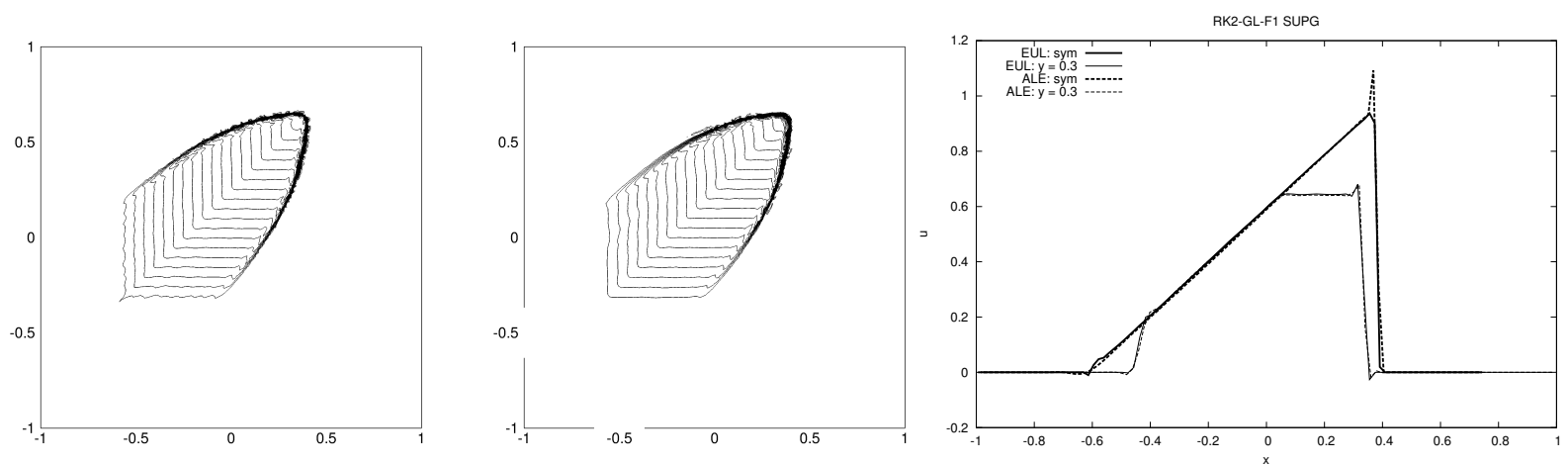

Figure 7: 2D Burger Equation, F1-GL SUPG scheme. Left and middle: 20 equispaced isolines between 0 and 1 at time $t=1$. left, Eulerian. right ALE. Bottom: comparison of the solution along the symmetry line and along the line $y=0.3$ 

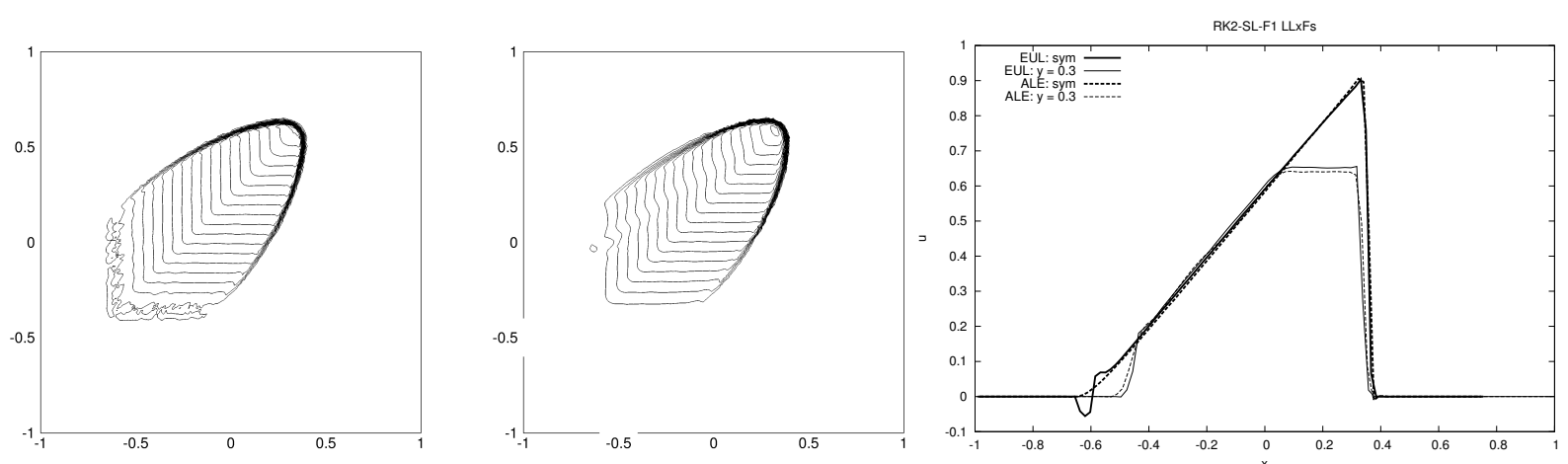

Figure 8: 2D Burger Equation, F1-SL LLxFs scheme. Left and middle: 20 equispaced isolines between 0 and 1 at time $t=1$. left, Eulerian. right ALE. Bottom: comparison of the solution along the symmetry line and along the line $y=0.3$
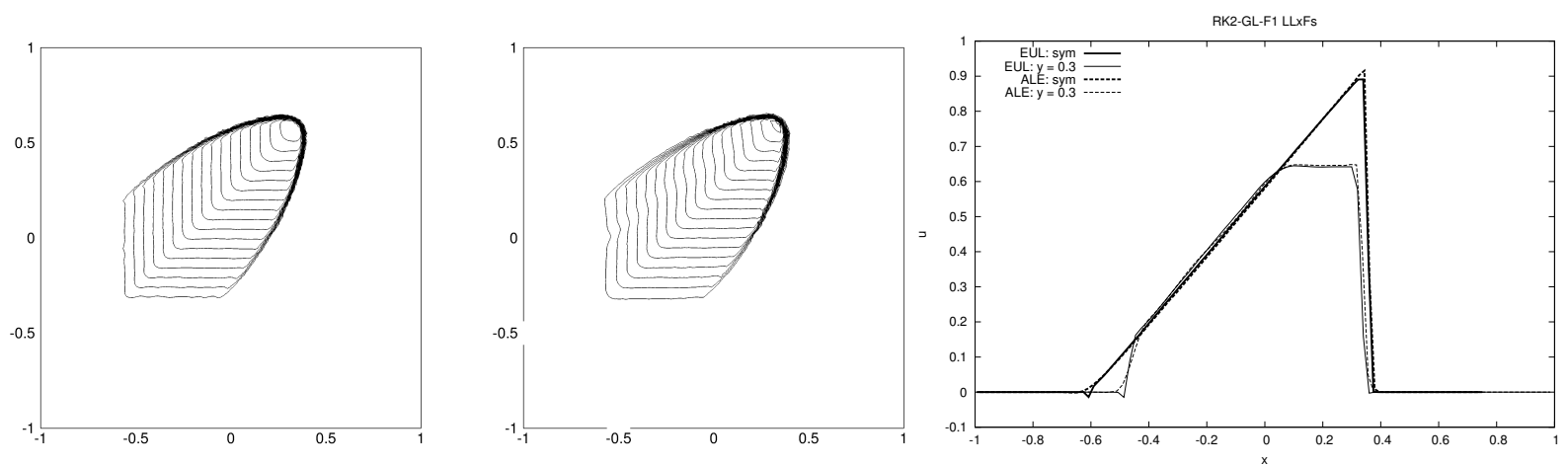

Figure 9: 2D Burger Equation, F1-GL LLxFs scheme. LEft and middle: 20 equispaced isolines between 0 and 1 at time $t=1$. left, Eulerian. right ALE. Right: comparison of the solution along the symmetry line and along the line $y=0.3$
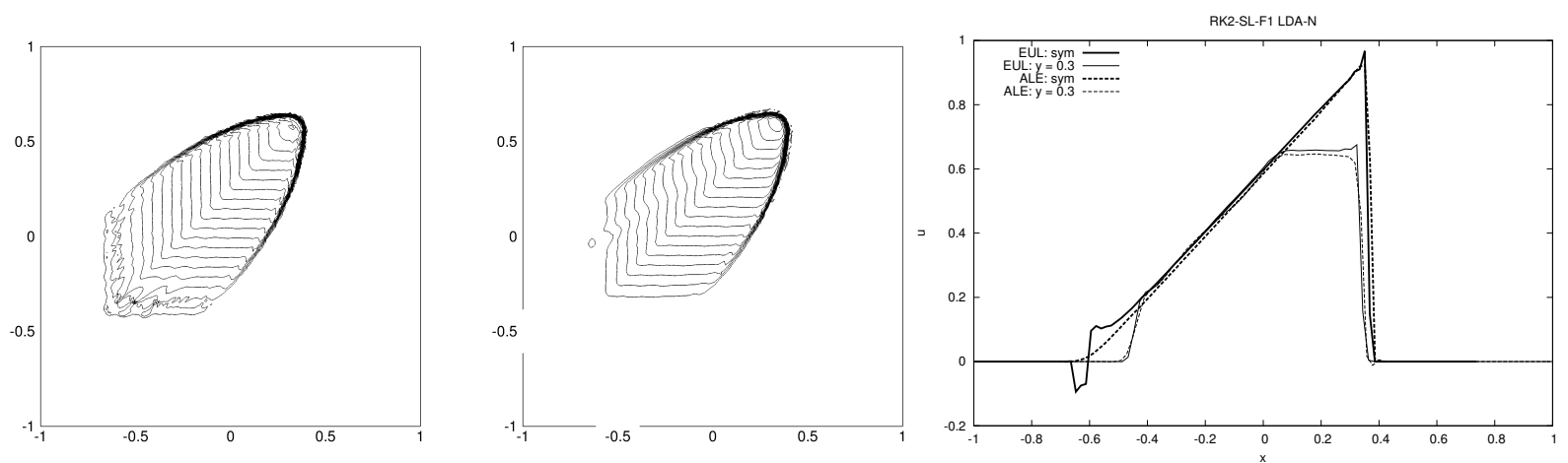

Figure 10: 2D Burger Equation, F1-SL LDA-N scheme. Left and middle: 20 equispaced isolines between 0 and 1 at time $t=1$. left, Eulerian. right ALE. Right: comparison of the solution along the symmetry line and along the line $y=0.3$ 

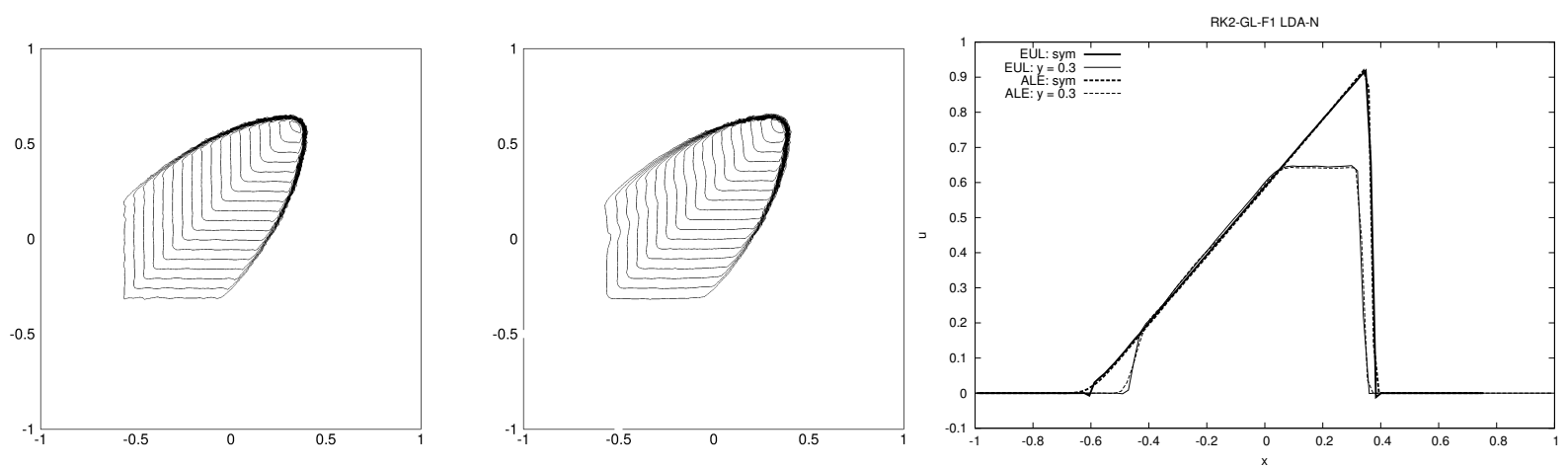

Figure 11: 2D Burger Equation, F1-GL LDA-N scheme. LEft and middle: 20 equispaced isolines between 0 and 1 at time $t=1$. left, Eulerian. right ALE. Right: comparison of the solution along the symmetry line and along the line $y=0.3$ 


\section{Application to the perfect gas Euler Equations}

In this section, we consider the applications of the ALE formulation proposed to the perfect gas Euler equations

$$
\frac{\partial \mathbf{u}}{\partial t}+\nabla \cdot \mathbf{f}=0 \quad \text { in } \Omega \in \mathbb{R}^{2}, t \in[0, T]
$$

with conserved variables and flux given by

$$
\mathbf{u}=\left[\begin{array}{c}
\rho \\
\rho u \\
\rho v \\
\rho E
\end{array}\right], \quad \mathbf{f}(\mathbf{u})=\left[\begin{array}{cc}
\rho u & \rho v \\
\rho u^{2}+p & \rho u v \\
\rho u v & \rho v^{2}+p \\
\rho H u & \rho H v
\end{array}\right]
$$

where $\rho$ is the fluid density, $\boldsymbol{u}=(u, v)$ is the flow speed, $E$ is the total energy per unit mass, $H$ is the total entalpy per unit mass

$$
H=E+\frac{p}{\rho}
$$

For polytropic ideal gas thermodynamic properties are completely defined by the following pair of equations of state

$$
p(T, \rho)=R T \rho, \quad e(T)=\frac{R T}{\gamma-1}
$$

where we have introduced the internal energy per unit mass $e$ and the temperature of the fluid $T$. Combining the two equations we obtain $p$ as a function of $e$ and $\rho$

$$
p(e, \rho)=(\gamma-1) \rho e
$$

The Euler equations are closed with the definition of internal energy

$$
e=E-\frac{1}{2}\|\boldsymbol{u}\|
$$

since we are able to express the pressure as function of the unknown $\rho, u, v, E$.

The Euler equations constitute a hyperbolic system, in particular given a vector $\boldsymbol{\xi} \in \mathbb{R}^{2}$, the flux Jacobian $\mathrm{K}(\mathrm{u}, \xi)=\frac{\partial \mathbf{f}}{\partial \mathrm{u}} \cdot \boldsymbol{\xi}$ admits a complete set of real eigenvalues and linearly independent eigenvectors. The eigenvalues of $\mathrm{K}(\mathbf{u}, \boldsymbol{\xi})$ are

$$
\lambda_{1,4}(\mathbf{u}, \boldsymbol{\xi})=\boldsymbol{u} \cdot \boldsymbol{\xi} \mp c(\mathbf{u})\|\boldsymbol{\xi}\|, \quad \lambda_{2,3}(\mathbf{u}, \boldsymbol{\xi})=\boldsymbol{u} \cdot \boldsymbol{\xi}
$$

where $c(\mathbf{u})=\sqrt{\gamma p / \rho}$ is the speed of sound.

\subsection{Implementation details}

We give in this section a few remarks on the implementation of the schemes for systems, the interested reader can consult [19, 30, 31, 10] for more details. The schemes presented in section §3.6, with the distribution strategies presented in section $\$ 1.4$ extend formally to hyperbolic systems of conservation laws, with the obvious change in dimensions for the residuals $\phi^{K}$ and $\Phi^{K}$ (cf. equations (1) and (50) which now are vectors, and for the upwind parameters $k_{i}$ which are now matrices $k_{i}=\mathbf{K}\left(\mathbf{u}, \boldsymbol{n}_{i}\right) / 2(\mathrm{cf}$. equation (36)). The sign and $(\cdot)^{+}$operators needed in the schemes are now computed by standard eigenvalue decomposition, while $\alpha^{K}$ in the $\mathrm{LxF}$ scheme is now replaced by the largest among the spectral radii of the $k_{j}$ matrices. Nonlinear schemes are implemented by using the same definitions of section 
$\S 1.4 .3$ only applied via a characteristic variable decomposition. For example, the blended scheme is now defined as

$$
\Phi_{i}^{L D A-N}=\sum_{m=1}^{4} \alpha_{m}^{L D A-N} \boldsymbol{r}_{m}
$$

where $\boldsymbol{r}_{m}$ is the $m$-th eigenvector of the matrix $\mathbf{K}(\mathbf{u}, \hat{\boldsymbol{u}})$, with $\hat{\boldsymbol{u}}$ the unit vector parallel to the flow speed, and with

$$
\alpha_{m}^{L D A-N}=(1-l) \varphi_{i m}^{L D A}+l \varphi_{i m}^{N} \quad \text { with } \quad l=\frac{\left|\varphi_{m}^{K}\right|}{\sum_{j \in K}\left|\varphi_{j m}^{N}\right|}
$$

with scalar projected quantities $\varphi$ obtained as

$$
\varphi_{m}=\ell_{m} \cdot \Phi
$$

with $\boldsymbol{\ell}_{m}$ the $m$-the left eigenvector of $\mathbf{K}(\mathbf{u}, \hat{\boldsymbol{u}})$. A similar projection is performed for the LLxF scheme, details are omitted for brevity. For the Euler equations, following [10], the LLxFs scheme (52) is replaced by the LLxF-SUPG scheme

$$
\Phi_{i}^{L L x F-S U P G}=(1-\delta) \Phi_{i}^{L L x F}+\delta \Phi_{i}^{S U P G}
$$

where the smoothness sensor is a scalar computed as (cf. equation (53))

$$
\delta\left(\mathbf{u}^{h}\right)=\min \left(1, \frac{1}{\frac{\left|\varphi_{s}^{K}\right|}{h_{K}^{2}}+\varepsilon}\right)
$$

where $\varphi_{s}^{K}$ is an approximation of the entropy residual computed as

$$
\varphi_{s}^{K}=\ell_{s} \cdot \Phi^{K} / \Delta t
$$

with $\ell_{s}$ the left eigenvector corresponding to the entropy wave.

\subsection{Numerical results}

We present three tests to show that the accuracy and robustness of the ALE discretization proposed. The first is a grid convergence study on the advection of a smooth constant density vortex. The second test case is a two-dimensional Riemann problem allowing to compare the Eulerian and ALE implementation on a complex non-smooth problem. The last test is a simple application involving a moving boundary.

For the first two tests, the following mapping used

$$
\left\{\begin{array}{l}
x=X+0.1 \sin (2 \pi X) \sin (2 \pi Y) \sin \left(2 \pi^{t} / t_{\max }\right) \\
y=Y+0.1 \sin (2 \pi X) \sin (2 \pi Y) \sin \left(2 \pi^{t} / t_{\max }\right)
\end{array}\right.
$$

All computations have been performed with a time step computed according to

$$
\Delta t=\mathrm{CFL} \min _{i \in \mathcal{T}_{h}} \frac{\left|S_{i}\right|}{\sum_{K \in \mathcal{D}_{i}} \alpha^{K}}
$$

with $\mathrm{CFL}=0.8$ 

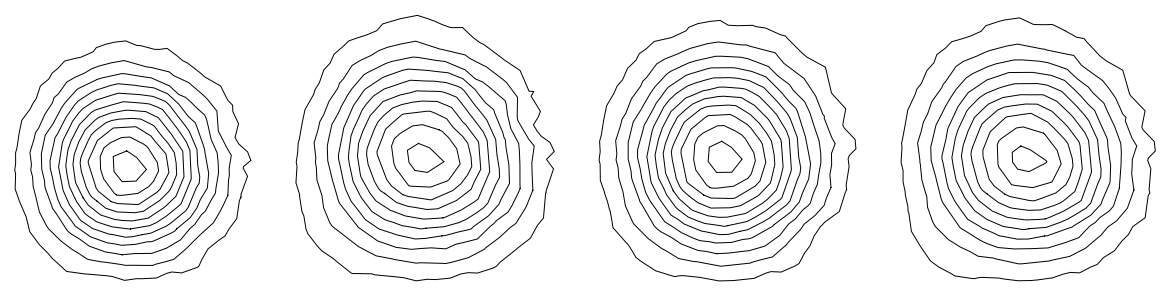

Figure 12: Advection of a vortex: 15 equispaced pressure isolines between level 94 and 101. From left to right : LDA (Eulerian), LDA (ALE), SUPG (Eulerian), and SUPG (ALE). All schemes based on the formulation F1 with selective lumping

\subsubsection{Advection of a Vortex}

The accuracy of the schemes is measured on the advection of a constant density vortex. The test case is the one used in [40. The flow velocity is given by the sum of a constant freestream velocity plus a circumferential perturbation

$$
\boldsymbol{u}_{0}=(6,0)+\Delta \boldsymbol{u}
$$

where, setting $x_{c}=x-0.5, y_{c}=y-0.5, \omega=15(\cos 4 \pi r+1)$ and $r=\sqrt{x_{c}^{2}+y_{c}^{2}}, \Delta \boldsymbol{u}=\mathbf{0}$ if $r \geq 0.25$, else

$$
\Delta \boldsymbol{u}=\left(-y_{c}, x_{c}\right) \omega
$$

Density is chosen constant $\rho_{0}=1.4$, the pressure is a given by

$$
\begin{gathered}
p_{0}=p_{m}+\Delta p \\
\Delta p=\frac{15^{2} \rho}{(4 \pi)^{2}}\left(2 \cos (4 \pi r)+8 \pi r \sin (4 \pi r)+\frac{\cos (8 \pi r)}{8}+\frac{4 \pi r \sin (8 \pi r)}{4}+12 \pi^{2} r^{2}\right)+C
\end{gathered}
$$

The constant $C$ is fixed such that the pressure at $r=0.25$ is the freestream pressure $p_{0}=p_{m}=100$. The maximum Mach number is $M_{0}^{\max }=0.8$.

The problem is solved on a square domain $[0,1] \times[0,1]$ until a final time $t_{\max }=1 / 6$. The domain is approximated with four unstructured triangulations with elements reference size $h \in\{1 / 40,1 / 80,1 / 160,1 / 320\}$.

In figure 13, we report a qualitative comparison of the pressure contour lines, showing that the ALE results closely follow the one in Eulerian framework. The grid convergence behavior is reported in figure 14. The convergence curves are qualitatively similar to the ones obtained for the scalar advection of a smooth profile. Second order of accuracy is achieved also in ALE framework for both the lumped and the selective formulation. The lowest convergence rate (equal to 1.5) is observed for the LDA-N scheme but, again, this is due to a switch to the first order $\mathrm{N}$ scheme in regions where strong gradients of the vortex are present. 

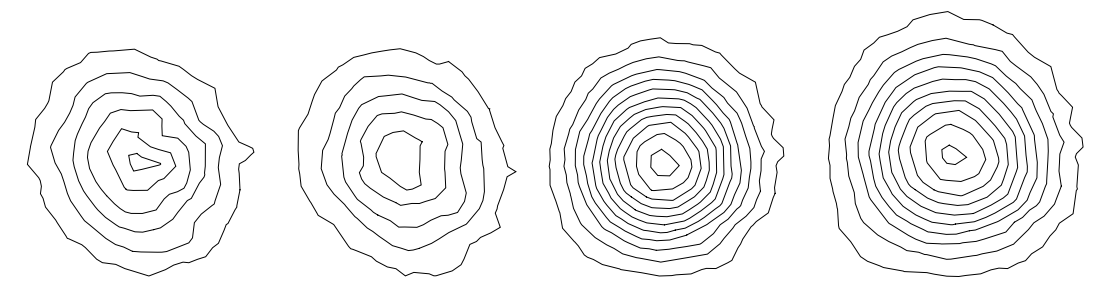

Figure 13: Advection of a vortex: 15 equispaced pressure isolines between level 94 and 101. From left to right : LDA-N (Eulerian), LDA-N (ALE), LLxF-SUPG (Eulerian), and LLxF-SUPG (ALE). All schemes based on the formulation F1 with selective lumping
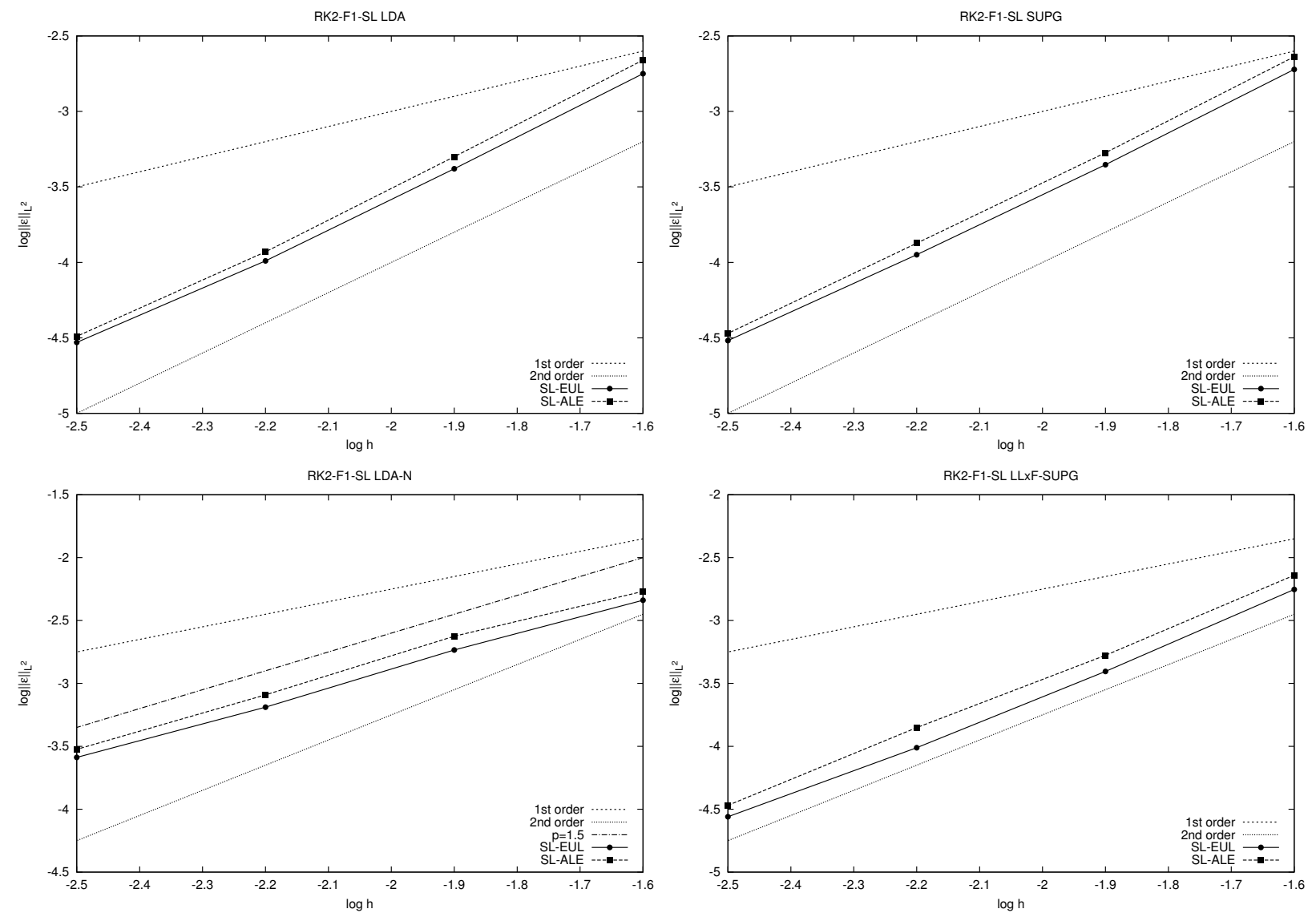

Figure 14: Advection of a vortex. Order of Convergence 


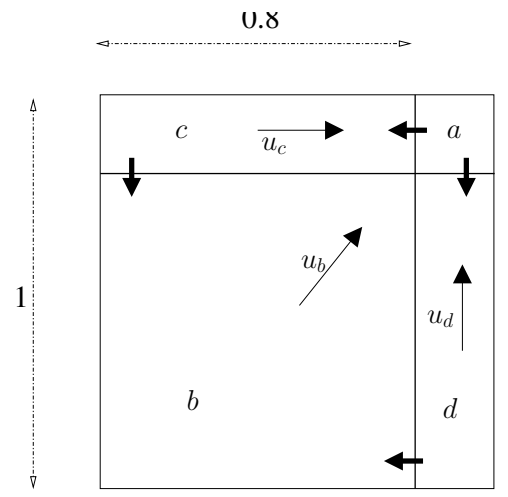

Figure 15: 2D Riemann Problem: initial solution

\subsubsection{A 2D Riemann problem}

This test case is contained in [17]. We use it to test the shock-capturing capabilities of the schemes. With reference to the notation of the figure 15 , the initial solution is given by

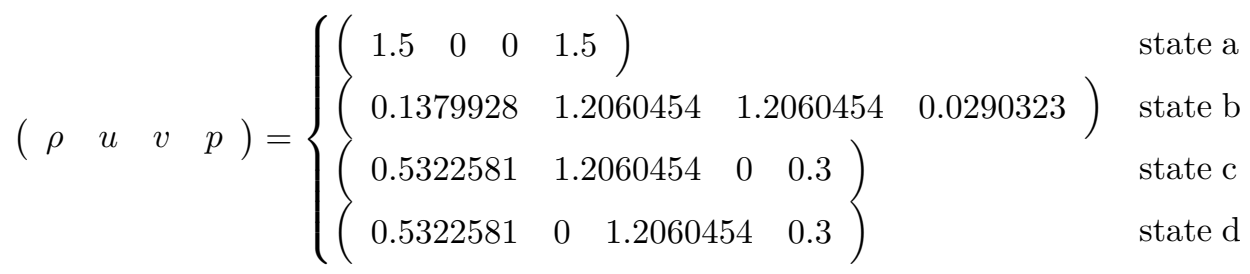

The structure of the solution is complex. Two normal shocks are interacting with two oblique shocks. This interaction generates two couples of symmetric lambda shocks with the appereance of contact discontinuities emanating from each of the 4 triple points. The amount of fluid that passes through the upper lambda shock structures (hence through two oblique shocks) is then pushed by the pressure gradient between state $a$ and $b$ into a transonic jet against the normal shock. The domain is a box $[0,1] \times[0,1]$ and it is approximated through a structured triangulation with element reference size $h=1 / 200$. The final time is $t_{\max }=0.8$.

Only the non-linear schemes are expected to give postive and second order accurate results, hence results in figure are referred only to the LDA-N and LLxF-SUPG schemes. The LDA-N case is shown in figure 16 17. The ALE results are overlapped, almost everywhere, with the ones obtained with Eulerian formulation on a fixed grid. As in that case, only when the global lumped formulation is used, we get positive results. With selective lumping, the solution is quite monotone but small oscillations appears near the discontinuities. 

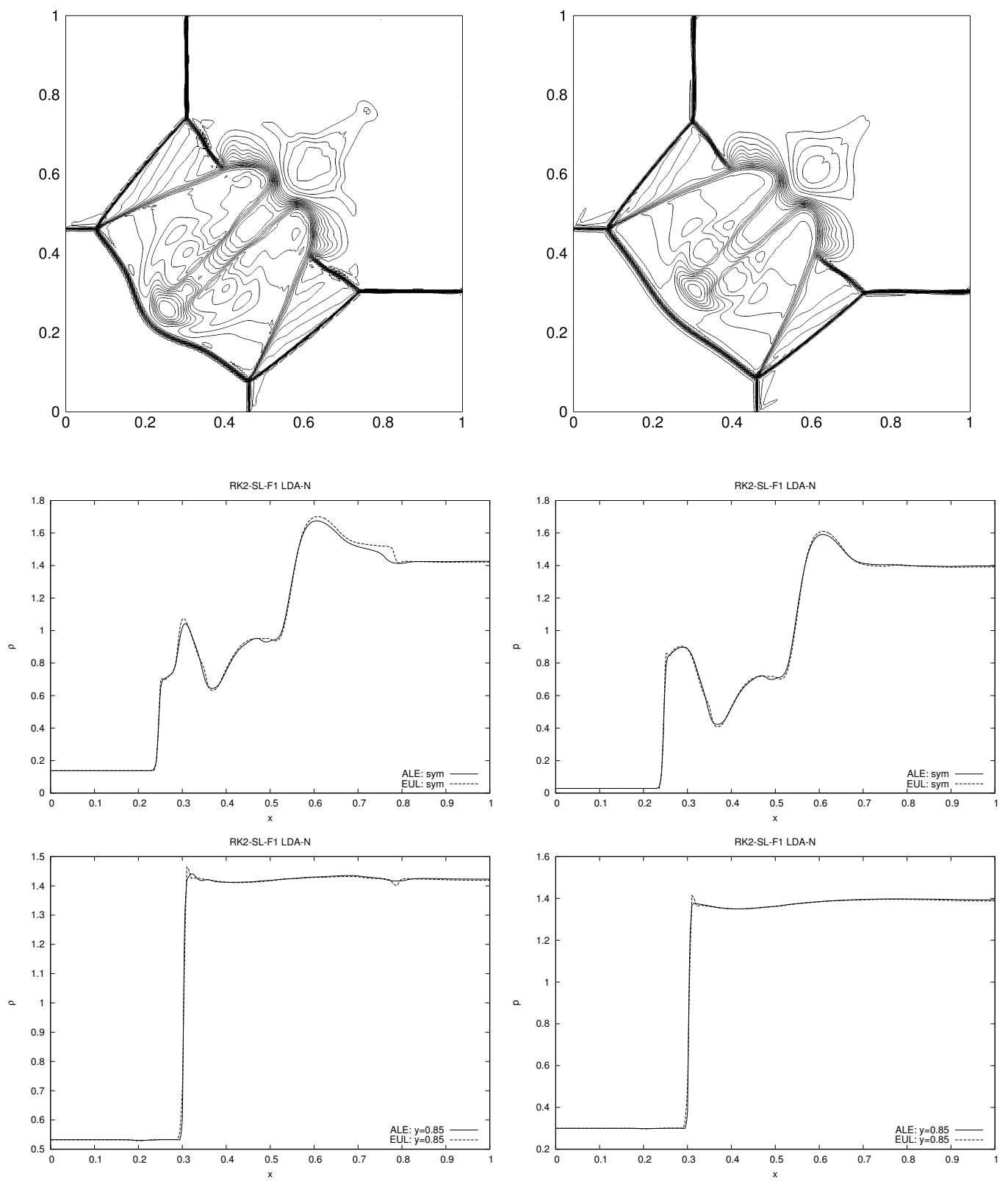

Figure 16: 2D Riemann problem computed with LDA-N scheme and RK2-F1-SL formulation. Top: 30 equispaced density isolines between maximum and minimum values of 1.65 and 0.1 . Top left: Eulerian formulation. Top right: ALE formulation. Middle: comparison of the solutions along the symmetry line. Bottom: comparison of the solutions at $y=0.85$ 

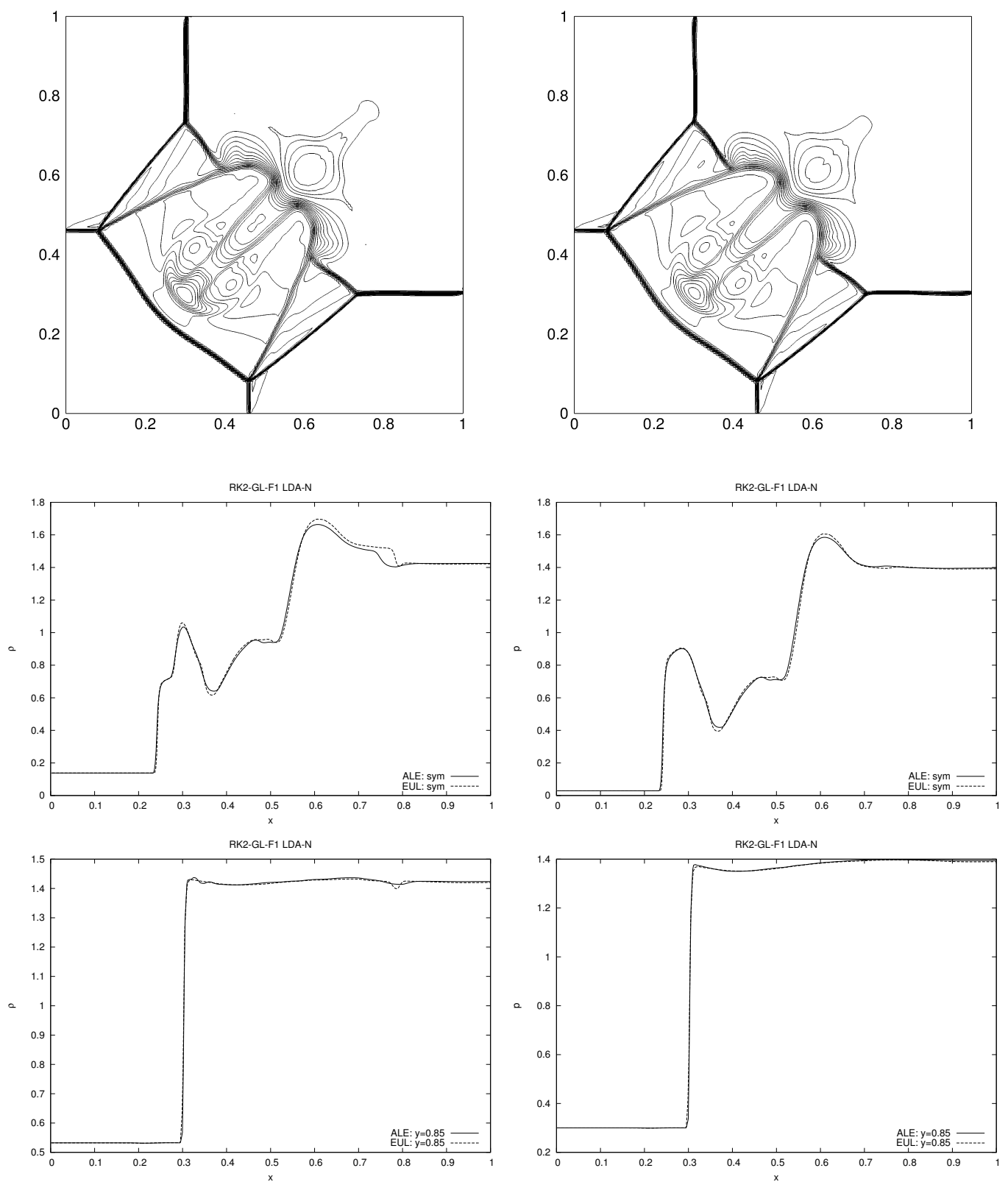

Figure 17: 2D Riemann problem computed with LDA-N scheme and RK2-F1-GL formulation. Top left: 35 equispaced density isolines for Eulerian formulation. Top right: 35 equispaced density isolines for ALE formulation. Middle: comparison of the solutions along the symmetry line. Bottom: comparison of the solutions at $y=0.85$ 

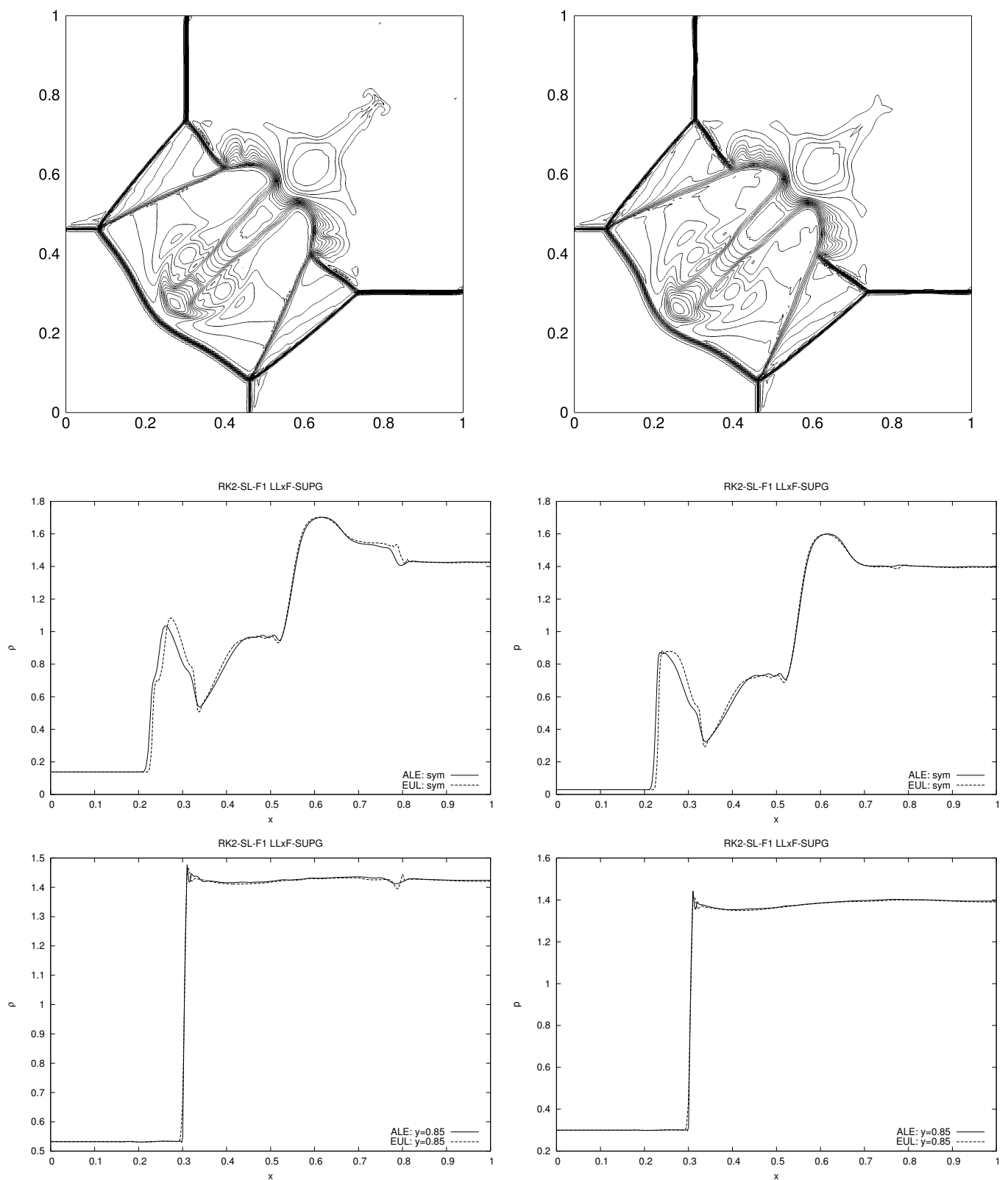

Figure 18: 2D Riemann problem computed with LLxF-SUPG scheme and RK2-F1-SL formulation. Top left: 35 equispaced density isolines for Eulerian formulation. Top right: 35 equispaced density isolines for ALE formulation. Middle: comparison of the solutions along the symmetry line. Bottom: comparison of the solutions at $y=0.85$ 

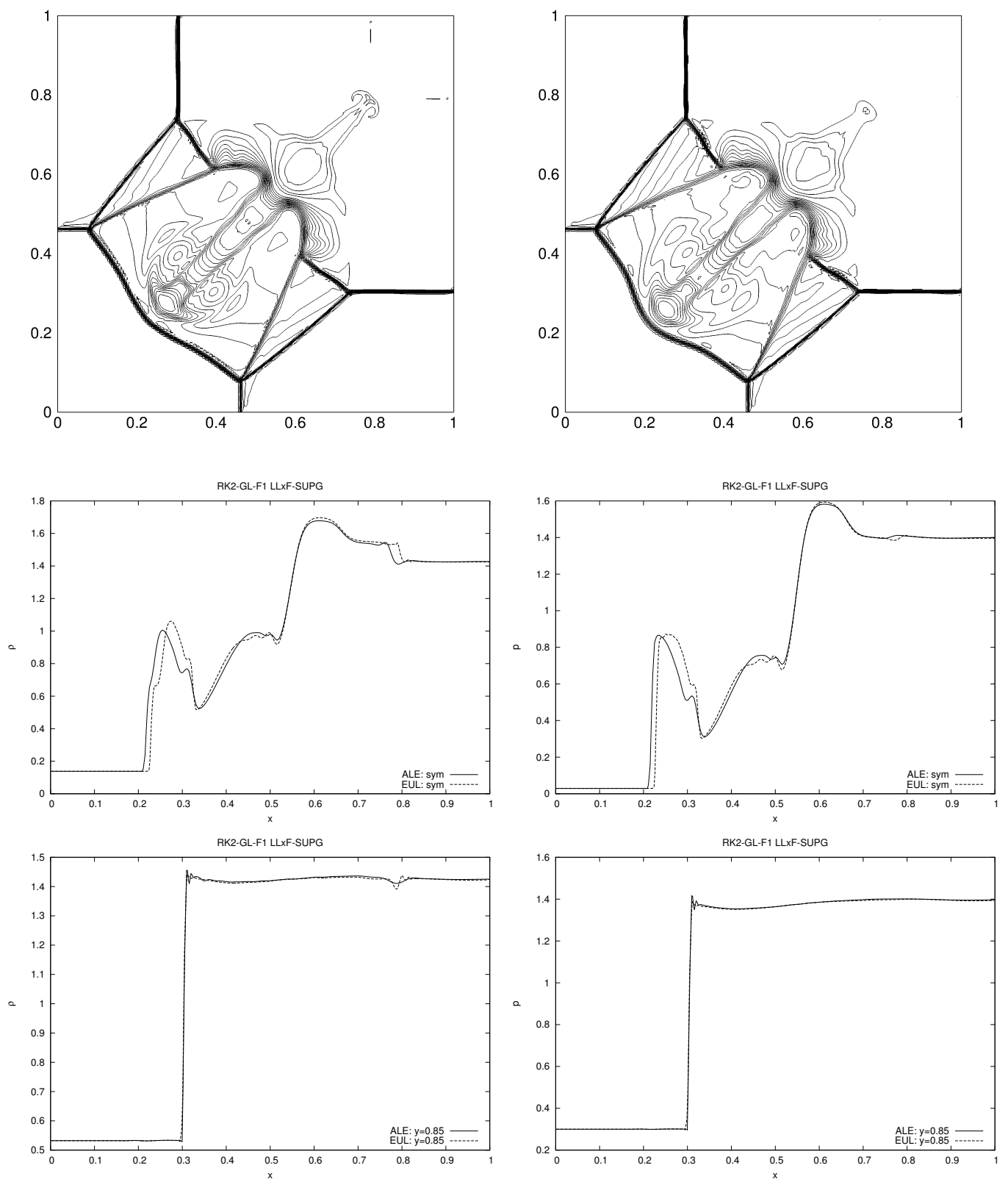

Figure 19: 2D Riemann problem computed with LLxF-SUPG scheme and RK2-F1-GL formulation. Top left: 35 equispaced density isolines for Eulerian formulation. Top right: 35 equispaced density isolines for ALE formulation. Middle: comparison of the solutions along the symmetry line. Bottom: comparison of the solutions at $y=0.85$ 

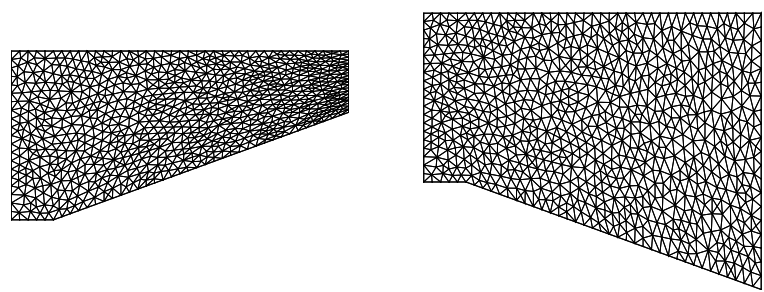

Figure 20: Topology for the grid. Left: compression. Right: expansion.

\subsubsection{An application: wind tunnel with wall deflection}

We have seen that, for all the test cases that we have run, the ALE formulation proposed here works well and we are able to recover almost the same result of Eulerian formulation. A very simple application, just to see the use of ALE formulation, is shown, involving moving boundaries. In this case Eulerian formulation cannot work without an interpolation step. The Eulerian formulation with the interpolation step has not been implemented, henco no comparison is given for this case.

We have a 2 D channel $[2 \times 1]$ with an hinge on the lower surface placed at $x=0.25$. This hinge allows a rigid deflection of the lower wall which is governed by the following exponential motion law for angle $\alpha$ defined from the horizontal axis

$$
\begin{cases}\alpha(t)=\alpha_{\max }\left(1-e^{-t / \tau}\right) & t \leq t_{\text {switch }} \\ \alpha(t)=\alpha_{\max }-2 \alpha_{\max }\left(1-e^{-\left(t-t_{\text {switch }}\right) / \tau}\right) & t>t_{\text {switch }}\end{cases}
$$

We choose the following values

$$
t_{\text {switch }}=1.25, \quad \tau=0.05, \quad \alpha_{\max }=20^{\circ}
$$

The final time for our simulation is $t_{\max }=2.5$. The domain is approximated with an unstructured triangulation with an element reference size $h=1 / 160$. During the simulation the grid is distorted solving a Laplace equation along every abscissa with boundary conditions given by the flap displacement at that abiscissa. In figure 20 the mapping for the grid is shown. Since shock waves are expected, we have tested only the non-linear schemes LDA-N and LLxF-SUPG. The formulation choosen is F1-GL. The Mach number at the inlet is $M=3$.

From the experiments we can observe that, after the transient, the shock structure finds a stable configuration close to the the analytical solution (Mach reflection of the shock at the upper surface) at $t \simeq 1.2$. Immediately after the wall deflects an unsteady interaction, between the shock and the expansion wave rising from the corner, is observed. The shock wave, while it is going back, takes an S-shaped configuration. In particular, in the region near the lower wall, the shock seems to be particularly strong becouse of the interaction between the accelerating flow, in expansion after the corner, and the compressed region at the outlet. Finally, at $t \simeq 2.5$, the supersonic Prandtl-Mayer expansion is recovered.

\section{Conclusion}

A novel method for the solution of hyperbolic equations in ALE framework has been presented in this paper. First conservation laws have been written in Arbitrary Lagrangian Eulerian formulation (ALE). Their derivation is addressed in section $§ 1$. 
$\mathrm{t}=\mathbf{0}$
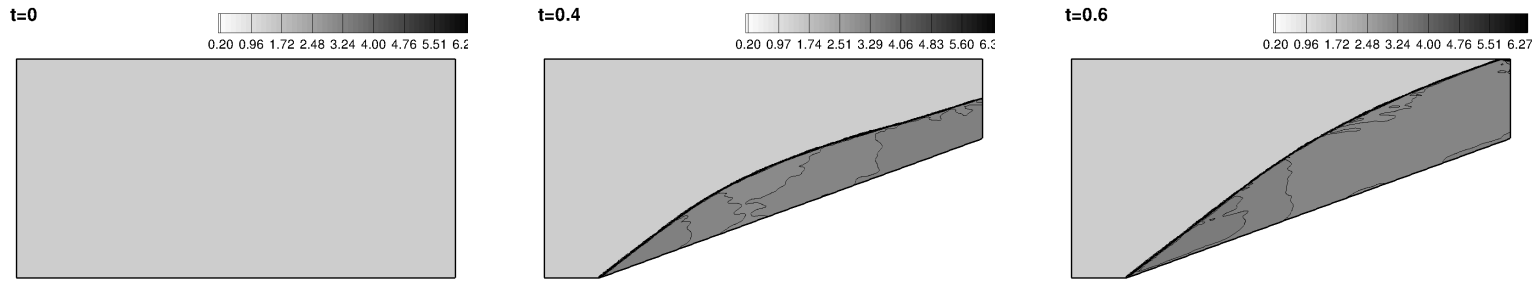

$t=0.0$

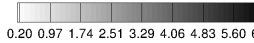
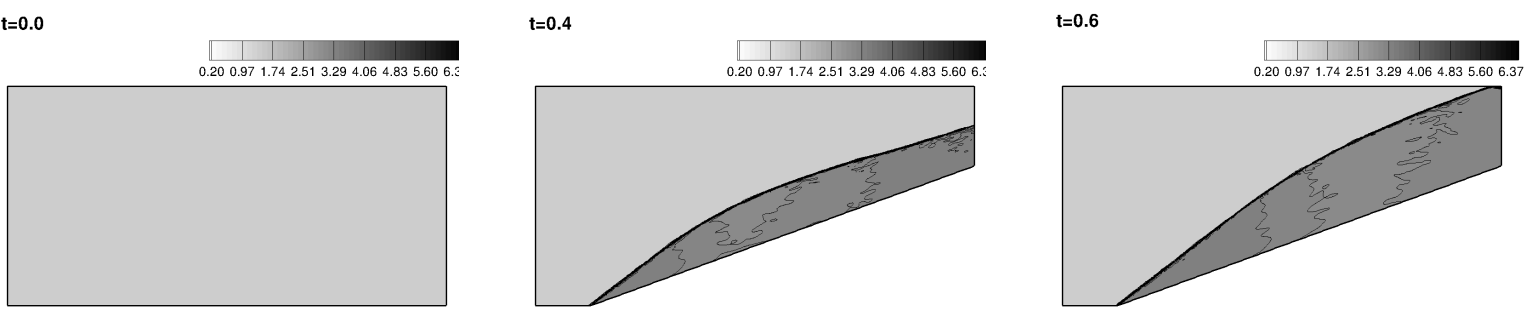

Figure 21: Mach 3 wind tunnel. Top row : LDA-N-F1-GL. Bottom row : LLxF-SUPG RK2-F1-GL. 50 equispaced density isolines between extreme values of $0.2-6.5$ at different time instants
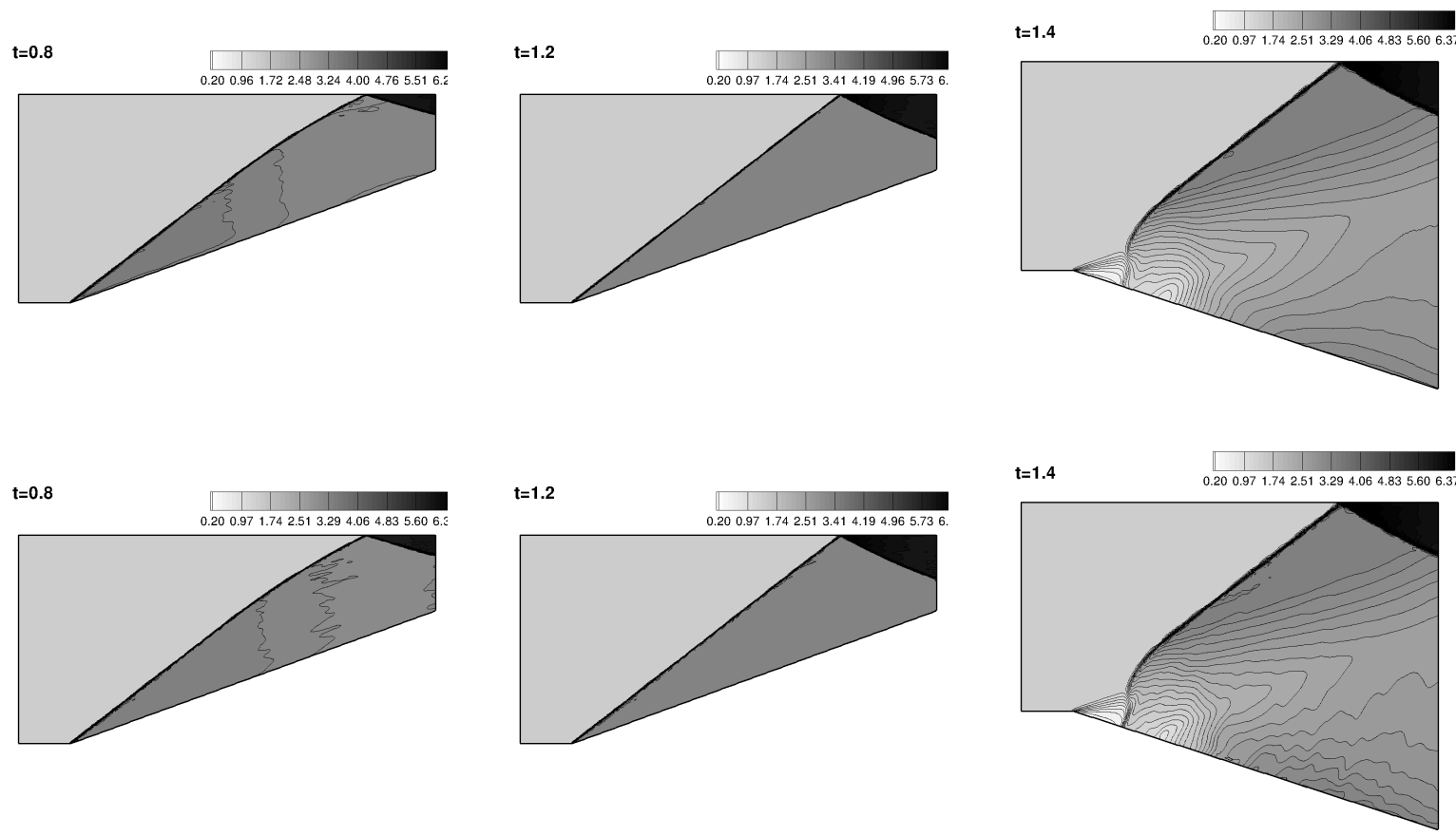

Figure 22: Mach 3 wind tunnel. Top row : LDA-N-F1-GL. Bottom row : LLxF-SUPG RK2-F1-GL. 50 equispaced density isolines between extreme values of $0.2-6.5$ at different time instants 

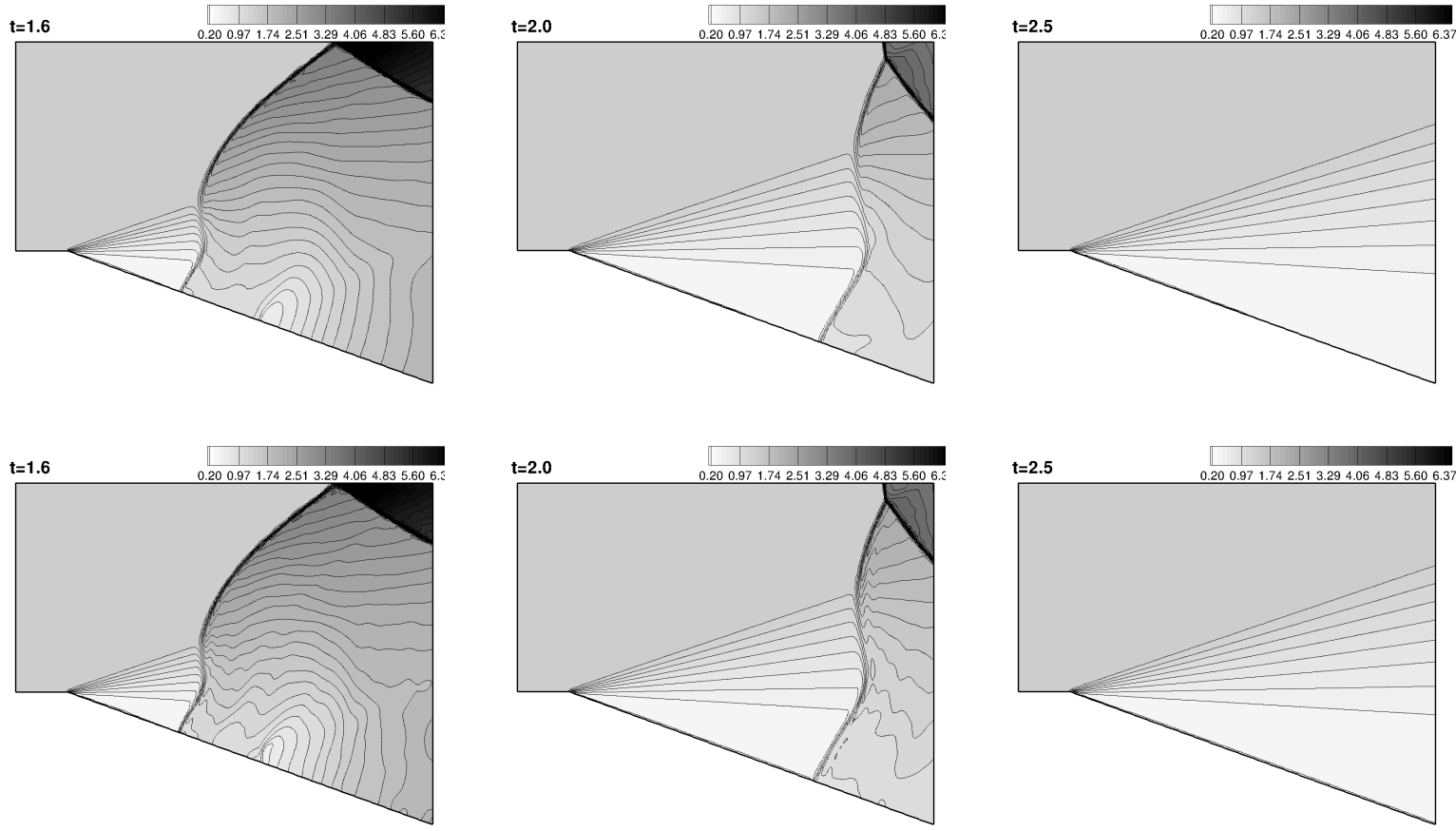

Figure 23: Mach 3 wind tunnel. Top row : LDA-N-F1-GL. Bottom row : LLxF-SUPG RK2-F1-GL. 50 equispaced density isolines between extreme values of $0.2-6.5$ at different time instants 
PDE are discretized in space with a Residual Distribution $(\mathcal{R D})$ approximation.For the sake of completness, we recall several properties of $\mathcal{R D}$. We also recall an explicit Runge Kutta 2 RD scheme on fixed grid for conservation laws written in Eulerian framework. We have extended the above scheme to equation in ALE form and we ended up with a scheme that results in minor modifications respect to the Eulerian algorithm. Particular emphasis is put on the Discrete Geometrical Conservation Law. .

The method has been studied extensively through theorical investigation and numerical experiments. Numerical results were in good agreement with Eulerian ones. The two advection test cases provides numerical evidence that convergence order is not spoiled when arbitrary grid distortions are involved, when the solution remains smooth. The Burger's equation test case and the Riemann problem showed the ability to handle well discontinuities.

Unfortunately in this paper we did not have the opportunity to cover some aspects that were not clear enough or that, in our opinion, deserve further studies, in particular it remains to analyse rigorously the positivity preserving propserties of this new scheme for scalar problems.

To conclude we mention possible future developments:

1. Grid adaptation not only to moving boundaries but also through a mechanism of node insertion/removal in order to refine the grid where strong gradients of the solution are expected. A succesfull algorithm has been already implemented by Guardone and Isola in a Finite Volume in 41 .

2. The extension to third order accurate solutions through high order space and time approximations. RD schemes that converges with order higher then two have been studied extensively for the steady case. The extension of the present work to third order should involve higher order elements and also an high order time discretization such as RK3.

\section{Acknowledgements}

RA is partially funded by the ERC Advanced Grant "ADECCO" (contract \#226316). We warmly thank Prof. A. Guardone for the enlightening discussions on mesh speeds in ALE computations.

\section{A Proof of proposition 4.1}

We start with the Selective Lumping case. For the first step $(k=1)$ assembling 4.5 (4.5) and at the same time using 79

$$
\begin{gathered}
\int_{\Omega^{n+1 / 2}} \varphi_{i} \frac{u_{h}^{1}-u_{h}^{n}}{\Delta t} d \boldsymbol{x}+\frac{\Delta t}{2} \int_{\Omega^{n+1 / 2}} \varphi_{i} \nabla \cdot \boldsymbol{\sigma}_{h}^{*}\left(u_{h}^{1}-u_{h}^{n}\right) d \boldsymbol{x}+ \\
+\int_{\Omega^{n+1 / 2}} w_{i}\left(\nabla \cdot f\left(u_{h}^{n}\right)-\boldsymbol{\sigma}_{h}^{*} \cdot \nabla u_{h}^{n}\right) d \boldsymbol{x}=0
\end{gathered}
$$

In a $\mathcal{R} \mathcal{D}$ formalism

$$
\sum_{K \in \mathcal{D}_{i}}\left(1+\nabla \cdot \boldsymbol{\sigma}_{h}^{*}\right) \frac{\left|K^{n+1 / 2}\right|}{3} \frac{u_{h}^{1}-u_{h}^{n}}{\Delta t}=-\sum_{K \in \mathcal{D}_{i}} \beta_{i} \sum_{j} k_{j}^{n} u_{j}^{n}=-\Delta t \sum_{K \in \mathcal{D}_{i}} \phi_{i}\left(u^{h}\right)
$$


Using the definition 82 , then the first line of $(84)$ is prooved.

For the second step $(k=2)$ the algebra is a little longer. We put in evidence the clear fact

$$
\sum_{K \in \mathcal{D}_{i}} \int_{K^{n+1 / 2}} \gamma_{i} \frac{\Delta \overline{u_{h}^{k}}}{\Delta t} d \boldsymbol{x}=\sum_{K \in \mathcal{D}_{i}} \int_{K^{n+1 / 2}} w_{i} \frac{\Delta \overline{u_{h}^{k}}}{\Delta t} d \boldsymbol{x}-\int_{\Omega^{n+1 / 2}} \varphi_{i} \frac{\Delta \overline{u_{h}^{k}}}{\Delta t} d \boldsymbol{x}
$$

Again assembling 4.54 4.5) and at the same time using (79)

$$
\begin{gathered}
\int_{\Omega^{n+1 / 2}} \varphi_{i} \frac{u_{h}^{n+1}-u_{h}^{n}}{\Delta t} d \boldsymbol{x}+\frac{\Delta t}{2} \int_{\Omega^{n+1 / 2}} \varphi_{i} \nabla \cdot \underline{\sigma}_{h}^{*} \frac{u_{h}^{n+1}+u_{h}^{n}}{\Delta t} d \boldsymbol{x}+ \\
-\frac{1}{2} \int_{\Omega^{n+1 / 2}} \varphi_{i} \nabla \cdot \boldsymbol{\sigma}_{h}^{*}\left(u_{h}^{1}+u_{h}^{n}\right) d \boldsymbol{x}-\int_{\Omega^{n+1 / 2}} \varphi_{i} \frac{u_{h}^{1}-u_{h}^{n}}{\Delta t} d \boldsymbol{x}+ \\
+\sum_{K \in \mathcal{D}_{i}} \int_{K^{n+1 / 2}} w_{i} \frac{u_{h}^{1}-u_{h}^{n}}{\Delta t} d \boldsymbol{x}+\frac{1}{2} \sum_{K \in \mathcal{D}_{i}} \int_{K^{n+1 / 2}} w_{i}\left(\nabla \cdot f\left(u_{h}^{n}\right)-\boldsymbol{\sigma}_{h}^{*} \cdot \nabla u_{h}^{n}\right) d \boldsymbol{x}+ \\
+\frac{1}{2} \sum_{K \in \mathcal{D}_{i}} \int_{K^{n+1 / 2}} w_{i}\left(\nabla \cdot f\left(u_{h}^{1}\right)-\boldsymbol{\sigma}_{h}^{*} \cdot \nabla u_{h}^{1}\right) d \boldsymbol{x}=0
\end{gathered}
$$

Now we sum and subtract the quantity $\frac{\Delta t}{2} \int_{\Omega^{n+1 / 2}} \varphi_{i} \nabla \cdot \boldsymbol{\sigma}_{h}^{*} \frac{u_{h}^{n+1}-u_{h}^{n}}{\Delta t} d \boldsymbol{x}$. The term with plus sum with the first term of the above equation, the term with minus sum with the second, the third and the fourth ones

$$
\begin{gathered}
\int_{\Omega^{n+1 / 2}}\left(1+\frac{\Delta t}{2} \nabla \cdot \underline{\sigma}_{h}^{*}\right) \varphi_{i} \frac{u_{h}^{n+1}-u_{h}^{n}}{\Delta t} d \boldsymbol{x}-\int_{\Omega^{n+1 / 2}}\left(1+\frac{\Delta t}{2} \nabla \cdot \boldsymbol{\sigma}_{h}^{*}\right) \varphi_{i} \frac{u_{h}^{1}-u_{h}^{n}}{\Delta t} d \boldsymbol{x} \\
+\sum_{K \in \mathcal{D}_{i}} \int_{K^{n+1 / 2}} w_{i} \frac{u_{h}^{1}-u_{h}^{n}}{\Delta t} d \boldsymbol{x}+\frac{1}{2} \sum_{K \in \mathcal{D}_{i}} \int_{K^{n+1 / 2}} w_{i}\left(\nabla \cdot \boldsymbol{f}\left(u_{h}^{n}\right)-\boldsymbol{\sigma}_{h}^{*} \cdot \nabla u_{h}^{n}\right) d \boldsymbol{x}+ \\
+\frac{1}{2} \sum_{K \in \mathcal{D}_{i}} \int_{K^{n+1 / 2}} w_{i}\left(\nabla \cdot \boldsymbol{f}\left(u_{h}^{1}\right)-\boldsymbol{\sigma}_{h}^{*} \cdot \nabla u_{h}^{1}\right) d \boldsymbol{x}=0
\end{gathered}
$$

The last three terms can be rewritten compactly with 4.5,

$$
\begin{gathered}
\int_{\Omega^{n+1 / 2}}\left(1+\frac{\Delta t}{2} \nabla \cdot \boldsymbol{\sigma}_{h}^{*}\right) \varphi_{i} \frac{u_{h}^{n+1}-u_{h}^{n}}{\Delta t} d \boldsymbol{x}= \\
=-\sum_{K \in \mathcal{D}_{i}} \Phi_{i}^{R K(2)}+\int_{\Omega^{n+1 / 2}}\left(1+\frac{\Delta t}{2} \nabla \cdot \boldsymbol{\sigma}_{h}^{*}\right) \varphi_{i} \frac{u_{h}^{1}-u_{h}^{n}}{\Delta t} d \boldsymbol{x}
\end{gathered}
$$

Developing both the modified mass matrices but lumping only the one on the right-hand side, then using definitions (82) and (85), (84) is finally proven.

Let us now consider the case of Global Lumping. The first step remain the same and has been already prooved

For the second step $(k=2)$ assembling 4.54 (4.5), togheter with 790,93

$$
\int_{\Omega^{n+1 / 2}} \varphi_{i} \frac{u_{h}^{n+1}-u_{h}^{n}}{\Delta t} d \boldsymbol{x}+\frac{\Delta t}{2} \int_{\Omega^{n+1 / 2}} \varphi_{i} \nabla \cdot \sigma_{h}^{*} \frac{u_{h}^{n+1}+u_{h}^{n}}{\Delta t} d \boldsymbol{x}+
$$




$$
\begin{gathered}
-\frac{1}{2} \int_{\Omega^{n+1 / 2}} \varphi_{i} \nabla \cdot \boldsymbol{\sigma}_{h}^{*}\left(u_{h}^{1}+u_{h}^{n}\right) d \boldsymbol{x}-\int_{\Omega^{n+1 / 2}} \varphi_{i} \frac{u_{h}^{1}-u_{h}^{n}}{\Delta t} d \underline{x}+ \\
+\sum_{K \in \mathcal{D}_{i}} \int_{K^{n+1 / 2}} w_{i} \frac{u_{h}^{1}-u_{h}^{n}}{\Delta t} d \boldsymbol{x}+\frac{1}{2} \sum_{K \in \mathcal{D}_{i}} \int_{K^{n+1 / 2}} w_{i}\left(\nabla \cdot \boldsymbol{f}\left(u_{h}^{n}\right)-\boldsymbol{\sigma}_{h}^{*} \cdot \nabla u_{h}^{n}\right) d \boldsymbol{x}+ \\
\frac{1}{2} \sum_{K \in \mathcal{D}_{i}} \int_{K^{n+1 / 2}} w_{i}\left(\nabla \cdot \boldsymbol{f}\left(u_{h}^{1}\right)-\boldsymbol{\sigma}_{h}^{*} \cdot \nabla u_{h}^{1}\right) d \boldsymbol{x}=0
\end{gathered}
$$

Summing the first and the fourth term togheter and the second and third too

$$
\begin{gathered}
\int_{\Omega^{n+1 / 2}} \varphi_{i} \frac{u_{h}^{n+1}-u_{h}^{1}}{\Delta t} d \boldsymbol{x}+\frac{\Delta t}{2} \int_{\Omega^{n+1 / 2}} \varphi_{i} \nabla \cdot \boldsymbol{\sigma}_{h}^{*} \frac{u_{h}^{n+1}-u_{h}^{1}}{\Delta t} d \boldsymbol{x}+ \\
+\sum_{K \in \mathcal{D}_{i}} \int_{K^{n+1 / 2}} w_{i} \frac{u_{h}^{1}-u_{h}^{n}}{\Delta t} d \boldsymbol{x}+\frac{1}{2} \sum_{K \in \mathcal{D}_{i}} \int_{K^{n+1 / 2}} w_{i}\left(\nabla \cdot \boldsymbol{f}\left(u_{h}^{n}\right)-\boldsymbol{\sigma}_{h}^{*} \cdot \nabla u_{h}^{n}\right) d \boldsymbol{x}+ \\
+\frac{1}{2} \sum_{K \in \mathcal{D}_{i}} \int_{K^{n+1 / 2}} w_{i}\left(\nabla \cdot \boldsymbol{f}\left(u_{h}^{1}\right)-\boldsymbol{\sigma}_{h}^{*} \cdot \nabla u_{h}^{1}\right) d \boldsymbol{x}=0
\end{gathered}
$$

The last three terms can be rewritten compactly with [4.5), while the first two terms sum up

$$
\int_{\Omega^{n+1 / 2}}\left(1+\frac{\Delta t}{2} \nabla \cdot \boldsymbol{\sigma}_{h}^{*}\right) \varphi_{i} \frac{u_{h}^{n+1}-u_{h}^{1}}{\Delta t} d \boldsymbol{x}=-\sum_{K \in \mathcal{D}_{i}} \Phi_{i}^{R K(2)}
$$

Developing the mass matrix, lumping it and using 82 we get the second line of 860 .

\section{References}

[1] J. Donea. Computational methods for transient analysis. chapter 10, Arbitrary Lagrangian Eulerian finite element methods. Elsevier Science Publisher, Amsterdam, 1983.

[2] C. Farhat, P.Geuzaine, and C. Grandmont. The discrete geometric conservation law and the nonlinaer stability of ale schemes for the solution of flow problems on moving grids. Journal of Computational Physics, 174(2):669-694, 2000.

[3] R.H. Ni. A multiple grid scheme for solving the euler equation. AIAA Journal, 20:1565-1571, 1981.

[4] J. Rice and R. Schnipke. A monotone streamline upwind method for convection dominated problems. Computer Methods in Applied Mechanics and Engineering, 48:313-327, 1985.

[5] A.N. Brooks and T.J.R.H ughes. Streamline upwind petrov-galerkin formulation for convection dominated flows with particular emphasis on the incompressible navier-stokes equations. Comp. Meth. Mech. Eng., 32:199-259, 1982.

[6] P.L. Roe. Fluctuations and signals - a framework for numerical evolution problems. In K.W. Morton and M.J. Baines, editors, Numerical Methods for Fluids Dynamics, pages 219-257. Academic Press, 1982.

[7] P. L. Roe. Linear advection schemes on triangular meshes. Technical Report CoA 8720, Cranfield Institute of Technology, 1987. 
[8] C. Michler and H. Deconinck. An arbitrary lagrangian eulerian formulation for residual distribution schemes on moving grids. Computers and Fluids, 32(1):59-71, 2001.

[9] J. Dobes. Numerical Algorithms for the Computation of Steady and Unsteady Compressible Flow over Moving Geometries - Application to Fluid-Structure Interaction. PhD thesis, Von Karman Institute, 2007.

[10] M. Ricchiuto and R. Abgrall. Explicit runge-kutta residual distribution schemes for time dependent problems: Second order case. Journal of Computational Physics, 229(16):5653 - 5691, 2010.

[11] J.-C. Carette, H. Deconinck, H. Paillère, and P.L. Roe. Multidimensional upwinding: its relation to finite elements. International Journal for Numerical Methods in Fluids, 20:935-955, 1995.

[12] J. Maerz and G. Degrez. Improving time accuracy of residual distribution schemes. Technical Report VKI-PR 96-17, von Karman Institute for Fluid Dynamics, 1996.

[13] A. Ferrante and H. Deconinck. Solution of the unsteady Euler equations using residual distribution and flux corrected transport. Technical Report VKI-PR 97-08, von Karman Institute for Fluid Dynamics, 1997.

[14] R. Abgrall and M. Mezine. Construction of second-order accurate monotone and stable residual distribution schemes for unsteady flow problems. J. Comput. Phys., 188:16-55, 2003.

[15] R. Abgrall and T.J. Barth. Residual distribution schemes for conservation laws via adaptive quadrature. SIAM J. Sci. Comput., 24(3):732-769, 2002.

[16] A. Csik, M. Ricchiuto, and H. Deconinck. A conservative formulation of the multidimensional upwind residual distribution schemes for general nonlinear conservation laws. J. Comput. Phys, $179(2): 286-312,2002$.

[17] M. Ricchiuto, A. Csik, and H.Deconinck. Residual distribution for general time-dependent conservation laws. J. Comput. Phys, 209(1):249-289, 2005.

[18] R. Struijs, H. Deconinck, P. De Palma, P.L. Roe, and K.G. Powell. Progress on multidimensional upwind Euler solvers for unstructured grids. AIAA paper 91-1550, 1991.

[19] R. Abgrall. Toward the ultimate conservative scheme : Following the quest. J. Comput. Phys, 167(2):277-315, 2001.

[20] S.M.J. Guzik and C.P.T. Groth. Comparison of solution accuracy of multidimensional residual distribution and godunov-type finite-volume methods. International Journal of Computational Fluid Dynamics, pages 61-83, 2008.

[21] S.P. Spekreijse. Multigrid solution of monotone second-order discretizations of hyperbolic conservation laws. Math. Comp., 49:135-155, 1987.

[22] T.J. Barth. Numerical methods for conservation laws on structured and unstructured meshes. VKI LS 2003-05, 33 $3^{\text {rd }}$ Computational Fluid dynamics Course, von Karman Institute for Fluid Dynamics, 2003.

[23] T.J. Barth and M. Ohlberger. Finite volume methods: foundation and analysis. In E. Stein, R. de Borst, and T.J.R. Hughes, editors, Encyclopedia of Computational Mechanics. John Wiley \& Sons, Ltd., 2004. 
[24] H. Deconinck and M. Ricchiuto. Residual distribution schemes: foundation and analysis. In E. Stein, R. de Borst, and T.J.R. Hughes, editors, Encyclopedia of Computational Mechanics. John Wiley \& Sons, Ltd., 2007. DOI: 10.1002/0470091355.ecm054.

[25] R. Abgrall and P.L. Roe. High-order fluctuation schemes on triangular meshes. J. Sci. Comput., 19(3):3-36, 2003.

[26] M. Ricchiuto, R. Abgrall, and H. Deconinck. Application of conservative residual distribution schemes to the solution of the shallow water equations on unstructured meshes,. J. Comput. Phys., 222:287-331, 2007.

[27] S. K. Godunov. A finite difference method for the numerical computation of discontinuous solutions of the equations of fluid dynamics. Mat. Sb., 47, 1959.

[28] H. Paillere and H. Deconinck. Compact cell vertex convection schemes on unstructured meshes. In H Deconinck and B Koren, editors, Notes on Numerical Fluid Mechanics, pages 1-50. Vieweg-Verlag, Braunschweig, Germany, 1997.

[29] H. Deconinck, K. Sermeus, and R. Abgrall. Status of multidimensional upwind residual distribution schemes and applications in aeronautics. AIAA paper 2000-2328, June 2000. AIAA CFD Conference, Denver (USA).

[30] R. Abgrall and M. Mezine. Construction of second-order accurate monotone and stable residual distribution schemes for steady flow problems. J. Comput. Phys., 195:474-507, 2004.

[31] R. Abgrall. Essentially non oscillatory residual distribution schemes for hyperbolic problems. J. Comput. Phys, 214(2):773-808, 2006.

[32] P. De Palma, G. Pascazio, G. Rossiello, and M. Napolitano. A second-order accurate monotone implicit fluctuation splitting scheme for unsteady problems. J. Comput. Phys, 208(1):1-33, 2005.

[33] M. Ricchiuto and A. Bollermann. Stabilized residual distribution for shallow water simulations. J. Comput. Phys, 228(4):1071-1115, 2009.

[34] M. Hubbard and M. Ricchiuto. Discontinuous upwind residual distribution: A route to unconditional positivity and high order accuracy. Computers and Fluids, 46(1):263 - 269, 2011.

[35] D. Sarmany, M. Hubbard, and M. Ricchiuto. Unconditionally stable space-time discontinuous residual distribution for shallow-water flows. Research Report RR-7958, INRIA, 2012. to appear on J.Comput.Phys.

[36] C. Bolley and M. Crouzeix. Conservation de la positivité lors de la discétization des problèmes d'èvolution paraboliques. R.A.I.R.O. Analyse Numèrique, 12:237-254, 1978.

[37] T. Wuilbaut and H. Deconinck. Improving monotonicity of the 2nd order backward difference time integration scheme by temporal limiting. In Haecheon Choi, HyongGwon Choi HyongGwon, and JungYul Yoo, editors, Computational Fluid Dynamics 2008, pages 733-738. Springer Berlin Heidelberg, 2009.

[38] G. Cohen, P. Joly, J.E. Roberts, and N. Tordjman. High order triangular finite elements with mass lumping for the wave equation. SIAM J. Numer. Anal., 38(6):2047-2078, 2001.

[39] M. Lesoinne and C. Farhat. Geometric conservation laws for flow problems with moving boundaries and deformable meshes, and their impact on aeroelastic computation. Computer methods in applied mechanics and engineering, 134(1-2):71-90, 1996. 
[40] J. Dobes and H. Deconinck. Second order blended multidimensional upwind residual distribution scheme for steady and unsteady computations. J.Comput.Appl.Math, 215(1):378-389, 2006.

[41] D. Isola. An Interpolation Free Two-Dimensional Conservative ALE scheme over Adaptive Unstructured Grids for Rotorcraf Aerodynamics. PhD thesis, Politecnico di Milano, Dipartimento di Ingegneria Aerospaziale, 2012. 


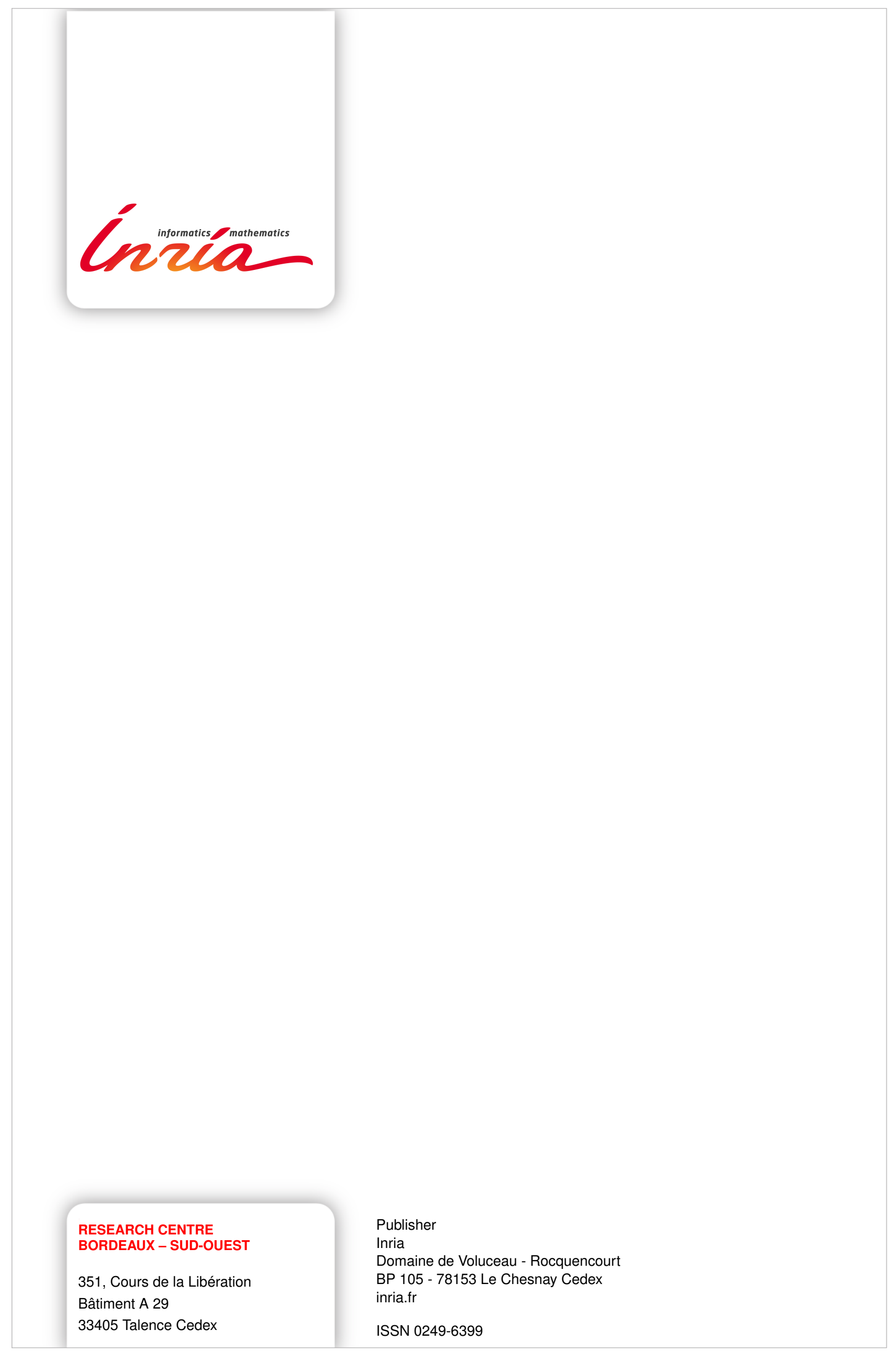

